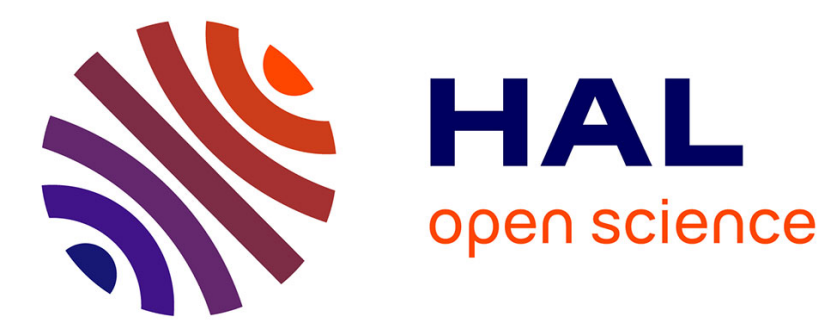

\title{
Interface structure of graphene on $\mathrm{SiC}:$ an ab initio and STM approach
}

Jean-Yves Veuillen, Fanny Hiebel, Laurence Magaud, Pierre Mallet, François

Varchon

\section{- To cite this version:}

Jean-Yves Veuillen, Fanny Hiebel, Laurence Magaud, Pierre Mallet, François Varchon. Interface structure of graphene on SiC: an ab initio and STM approach. Journal of Physics D: Applied Physics, 2010, 43 (37), pp.374008. 10.1088/0022-3727/43/37/374008 . hal-00569698

\section{HAL Id: hal-00569698 \\ https://hal.science/hal-00569698}

Submitted on 25 Feb 2011

HAL is a multi-disciplinary open access archive for the deposit and dissemination of scientific research documents, whether they are published or not. The documents may come from teaching and research institutions in France or abroad, or from public or private research centers.
L'archive ouverte pluridisciplinaire HAL, est destinée au dépôt et à la diffusion de documents scientifiques de niveau recherche, publiés ou non, émanant des établissements d'enseignement et de recherche français ou étrangers, des laboratoires publics ou privés. 


\title{
Interface structure of graphene on $\mathrm{SiC}:$ an ab initio and $\mathrm{STM}$ approach
}

\author{
J-Y Veuillen ${ }^{1, *}$, F Hiebel $^{1}$, L Magaud $^{1}$, P Mallet $^{1}$ and F Varchon ${ }^{1,2}$ \\ ${ }^{1}$ Institut Néel, CNRS-UJF, Boîte Postale 166, 38042 Grenoble, France \\ ${ }^{2}$ European Theoretical Spectroscopy Facility, Unité PCPM, Université catholique de \\ Louvain, Place Croix du Sud 1, 1348 Louvain-la-Neuve, Belgium \\ ${ }^{*}$ Corresponding author
}

E-mails : jean-yves.veuillen@grenoble.cnrs.fr, fanny.hiebel@grenoble.cnrs.fr, laurence.magaud@grenoble.cnrs.fr, pierre.mallet@grenoble.cnrs.fr, francois.varchon@uclouvain.be

\begin{abstract}
High temperature treatment of $\mathrm{SiC}$ surfaces is a well established technique for producing graphene directly on top of an insulating substrate. In this domain an important question is the influence of the substrate on the atomic and electronic structure of the graphene layers. This requires a detailed investigation of the interactions at the graphene-SiC interface. Surface science techniques and ab initio calculations are well suited for that purpose. In this article, we present a brief review of the recent investigations performed in this domain by scanning tunnelling microscopy (STM) and ab initio simulations. It is largely based on the work performed in our group, but it also provides a survey of the literature in these fields. Both the so-called $\mathrm{Si}$ and $\mathrm{C}$ face of the hexagonal $6 \mathrm{H}(4 \mathrm{H}) \mathrm{SiC}\{0001\}$ substrates will be considered, as they show markedly different behaviour.
\end{abstract}

PACS numbers : 73.20._r, 73.22.Pr, 81.05.ue, 68.37.Ef, 68.65.Pq, 71.15.Mb

\section{Introduction}

Graphene is the name given to a single atomic plane of graphite, or equivalently to a twodimensional (2D) system made of carbon atoms which form a honeycomb structure. Although this material has been studied theoretically for more than sixty years [1], the measurement of the physical properties of graphene had to wait for the development of suitable fabrication techniques [2,3]. Soon afterwards outstanding transport properties -high mobility, long phase coherence length or chiral quantum Hall effect- were reported [4-6]. This has triggered an enormous activity on this topic, aimed 
either at understanding the basic physical properties of the material or at looking for applications of this original 2D system, especially in the field of micro/nano electronics (for reviews see [7-9]).

Various strategies exist for preparing graphene samples. The popular micromechanical exfoliation technique [2] produces flakes with typical size of the order of $10 \mu \mathrm{m}$ [7], lying on an insulating substrate and which can thus be gated [7]. Chemical vapour deposition (CVD) techniques yield large size $(\approx \mathrm{cm})$ graphene layers on metallic substrates (see e. g. $[10,11])$. The transfer of such CVD grown films on insulating substrates can be achieved after etching away the underlying metal substrate [11]. The technique we focus on in this report is the high temperature decomposition of $\mathrm{SiC}$ crystals [3]. It can in principle lead to wafer sized samples directly formed on an insulating substrate. The large sample size is an advantage not only in the perspective of large scale device fabrication but also for characterizing the system with "wide beam" techniques such as optical spectroscopy [12], angle resolved photoemission (ARPES) [13,14] or X-ray diffraction [15]. The insulating substrate allows the straight realisation of transport experiments and of electrical devices in the graphene layer (semi-insulating $\mathrm{SiC}$ wafers are available, although they are quite expensive). One major issue -for all preparation techniques- is to control the homogeneity of the graphene thickness across the whole sample and to maximize the size of the (graphene) domains. For the process we consider here $-i$. e. the high temperature decomposition of $\mathrm{SiC}$ crystals- the growth of homogeneous graphene samples with coherent domains larger than 1 micrometre has been demonstrated recently [48, 49, 51, 52, 92, 93].

Graphitization of polar faces of $\mathrm{SiC}$ substrates upon high temperature treatment has long been known [16]. The films formed in this way are usually called few layers graphene (FLG), since carbon films with different thickness can be formed depending on the temperature and duration of the thermal treatment [16-18]. The mechanism leading to the formation of the FLG is thought to be the preferential sublimation of silicon atoms from the $\mathrm{SiC}$ substrate, followed by a collapse of the excess surface $\mathrm{C}$ atoms to form the graphitic film [16-19]. Early experiments using surface science techniques have revealed that FLG showed the structural and electronic signatures of graphitic layers [16-18, 20]. More recently the group of Walt de Heer has demonstrated that this technique could yield high quality samples with physical properties similar to those expected for ideal graphene [3, 6, 8, 12]. This has led to a renewal of interest for this system in the surface science community. Modern spectroscopy and microscopy techniques indeed allow the analysis of the structural and electronic properties of this material with an unprecedented level of resolution.

In this article we shall present a review of the recent contribution of scanning tunneling microscopy (STM) and of ab initio calculations to the understanding of the properties of FLG on SiC. It is largely based on the work performed in our group, but it also provides a survey of the literature in these fields. We shall focus on the early stages of FLG growth; this is on monolayer (ML) or bilayer (BL) graphene samples. In this thickness range one important issue is the possible influence of the substrate on the atomic or electronic structure of the graphene layers. We shall therefore pay particular 
attention to the nature of the interface between FLG and the SiC surface. It will be shown that the interaction with the substrate generates soft ripples in the graphene layer. This substrate induced warping has a different origin from the spontaneous rippling which would stabilize the -otherwise unstable- free standing graphene $[7,9]$.

The paper is organised as follows. In the first section we shall give a brief account of the structure and electronic properties of ideal graphene. We shall also summarize the basic properties of the substrate surfaces and we shall give a brief introduction to the experimental techniques. In a second part we shall consider the work made on the mono and bilayer graphene formed on the socalled Si face of the substrate. The third part is devoted to the investigations made in the monolayer (ML) range on the so-called " $\mathrm{C}$-face" of the substrate.

\section{Basic presentation of the material and of the techniques}

\subsection{Electronic structure of ideal graphene}

The atomic structure of ideal graphene is shown in figure 1-a. This 2D crystal has a hexagonal Bravais lattice with two $\mathrm{C}$ atoms (labelled A and B) per unit cell. This gives rise to a honeycomb structure composed of $\mathrm{C}$ hexagons. In this material, $\mathrm{C}$ atoms are in the $\mathrm{sp}^{2}$ hybridisation state, with one $\mathrm{p}_{z}$ orbital -pointing perpendicular to the crystal plane- per atom. The $\mathrm{sp}^{2}$ orbitals on neighbouring $\mathrm{C}$ atoms form strong $\sigma$ (in-plane) covalent bonds. $\pi$ bonds are formed by the lateral overlap of the remaining $\mathrm{p}_{z}$ orbitals.

The band structure of ideal graphene, obtained from ab initio calculations [21], is shown in figure 1-c. It is shown along specific directions of the 2D Brillouin zone (BZ) depicted in figure 1-d. Two points called $\mathrm{K}$ and $\mathrm{K}$, located at the corner of the BZ, are of particular importance as shown below. The band structure (figure 1-c) shows a set of three $\sigma$ bands located rather far in energy (more than $3 \mathrm{eV}$ ) below the Fermi level. The low energy electronic states are derived from the $\pi / \pi^{*}$ bands. These bands touch only at the K and K' points of the BZ. Around these points, the $\pi / \pi^{*}$ bands have a linear and isotropic dispersion in an energy range of approximately $\pm 0.5 \mathrm{eV}$, with a Fermi velocity $\mathrm{v}_{\mathrm{F}} \approx 10^{6} \mathrm{~m} / \mathrm{s}$. A sketch of the low energy band structure $\mathrm{E}(\mathbf{q})-\mathbf{q}$ being the wave vector measured from the $\mathrm{K} / \mathrm{K}$ ' points- close to the $\mathrm{K}$ (or $\mathrm{K}$ ') point is shown in figure 1-e. This structure is called the "Dirac cone" in the literature, and the point where $\pi$ and $\pi^{*}$ bands meet is called the Dirac point (its energy is noted $E_{D}$ afterwards). Consequently, the density of state as a function of energy is linear close to $E_{D}$, being zero at the Dirac point. For neutral (undoped) graphene the Fermi energy is at the Dirac point since the $\pi$ and $\sigma$ bands are fully occupied whereas the $\pi^{*}$ and $\sigma^{*}$ bands are empty. The isoenergy contour computed at $+0.5 \mathrm{eV}$ above the Dirac point is shown in figure 1-d (black lines). It reveals tiny pockets centred at the BZ corner. They show a slight deviation from the circular shape known as "trigonal warping". The band structure of figure 1-c has been reported by many groups. It has been 
computed for the first time in 1947 [1] using a tight-binding technique. It turns out that a simple tight binding technique including only the $\mathrm{p}_{z}$ orbital and a nearest neighbour interaction is sufficient to describe the important features of the band structure close to the Dirac point [10].

Apart from the linear dispersion, another fundamental characteristics of the low energy electronic states of graphene -i. e states close to the Dirac point- is the so-called "electronic chirality" $[10,22]$. It results from a specific symmetry of the electron wave-functions, which originates from the presence of two identical (C) atoms per unit cell. This property can be understood in the simple tight binding scheme quoted above [10, 23]. For a given wave vector $\mathbf{k}$, the Bloch wavefunctions $\Psi_{\mathbf{k}}(\mathbf{r})$ of the low energy states can be decomposed on a basis $\left\{\Psi_{\mathbf{k}}^{\mathrm{A}}(\mathbf{r}), \Psi_{\mathbf{k}}^{\mathrm{B}}(\mathbf{r})\right\}$, where $\Psi_{\mathbf{k}}^{\mathrm{A}}(\mathbf{r})\left(\Psi_{\mathbf{k}}^{\mathrm{B}}(\mathbf{r})\right)$ is a linear combination of $\mathrm{p}_{z}$ states centred on $\mathrm{C}$ atoms which belong to the $\mathrm{A}(\mathrm{B})$ sublattice (see [23] for details):

$\Psi_{\mathbf{k}}(\mathbf{r})=\mathrm{C}_{\mathrm{A}}(\mathbf{k}) \Psi_{\mathbf{k}}^{\mathrm{A}}(\mathbf{r})+\mathrm{C}_{\mathrm{B}}(\mathbf{k}) \Psi_{\mathbf{k}}^{\mathrm{B}}(\mathbf{r})$

One has: $\left|C_{A}(\mathbf{k})\right|=\left|C_{B}(\mathbf{k})\right|$, which means that for any eigenstate (and thus any energy) the Local Density of States (LDOS) should be identical on the A and on the B atoms.

For states close to the Dirac points at $K\left(K^{\prime}\right)$, one can write: $\mathbf{k}=\Gamma \mathbf{K}+\mathbf{q}\left(\mathbf{k}=\Gamma K^{\prime}+\mathbf{q}\right)$, where $\mathbf{q}$ is the wave vector measured from the $\mathrm{K}\left(\mathrm{K}^{\prime}\right)$ point in the corresponding valley. Performing a low energy expansion to the first order in $|\mathbf{q}|$ one obtains the linear and isotropic band dispersion in the vicinity of the Dirac point(s). Moreover, with a proper choice of the basis (and of the axis), see [23], a simple relationship between $C_{B}$ and $C_{A}$ is found. For example, for states with energy $E>E_{D}$ (electron states) in the vicinity of the $\mathrm{K}$ point:

$\mathrm{C}_{\mathrm{B}}(\mathbf{q})=\mathrm{C}_{\mathrm{A}}(\mathbf{q}) \mathrm{e}^{\mathrm{i} \theta}$

where $\theta$ is the angle between the $\mathbf{q}$ vector and the $\mathrm{x}$ axis. Similar relations are found for hole states $(\mathrm{E}<$ $E_{D}$ ) and in the other valley (close to the $K^{\prime}$ point). Equation (2) implies that $C_{B}$ changes sign (with respect to $\mathrm{C}_{\mathrm{A}}$ ) when $\mathbf{q}$ is changed into $-\mathbf{q}$ in the valley at $\mathrm{K}$, which occurs in the case of intravalley backscattering (the same is true in the valley at K'). This leads to the so-called absence of backscattering by long range scatterers [24] and for $\mathrm{AB}$ symmetric potentials.

The notions of pseudo-spin and of electronic chirality are related to the symmetry property of the wavefunction given by Equation (2) [10]. The (electronic) chirality manifests in several outstanding properties of graphene such as the chiral quantum Hall effect [4, 5], the Klein tunnelling $[22,25,26]$ or the weak antilocalisation [27-29]. It is thus a fundamental property of the material.

\subsection{Ideal bilayer graphene}

The structure of bilayer graphene is depicted in figure 1-b. It is shown in the so-called "Bernal stacking" which is usually found in graphite. In the top layer, the "A type" atom is located right above a "B type" atom in the bottom layer. The "B type" atom in the top layer is located above a hexagonal "hollow" site of the bottom layer. The (weak) inter-plane interaction (similar to the one in graphite) 
breaks the equivalency between the A and B atoms in both layers. The computed LDOS is different at low energy (in the range $E_{D} \pm \gamma_{1}$, where $\gamma_{1}$ is the interlayer hopping parameter) on the $A$ and $B$ sublattices within each layer [30]. The LDOS is larger on the B site in this energy range. For energies larger than $E_{D}+\gamma_{1}\left(\right.$ smaller than $\left.E_{D^{-}} \gamma_{1}\right)$ the LDOS on A and B sublattices becomes similar. The typical value of $\gamma_{1}$ is $0.4 \mathrm{eV}$ [30].

The electronic structure of bilayer graphene in Bernal stacking is fairly different from the one of (monolayer) graphene (for a review see [31]). The bands have a parabolic dispersion at low energy, and the electronic chirality is different from the one found in the monolayer. As a consequence, backscattering is no longer forbidden in this system. Moreover a gap can open at the Dirac point for an asymmetric bilayer, that is for different on-site energies on the top and bottom layers. This point will be considered in section 2 for the case of epitaxial layer.

Notice that the results summarized in this sub-section only apply to the case of a Bernal stacked bilayer and that they should not be generalized to an arbitrary stacking (see section 3).

\subsection{SiC substrate surfaces and graphitization techniques}

Graphitisation is usually performed on the polar faces of commercial hexagonal $4 \mathrm{H}$ or $6 \mathrm{H}-\mathrm{SiC}$ wafers. To our knowledge, no significant differences have been found between these two different polytypes for the purpose of the discussion in this manuscript. Due to the polar nature of the $\mathrm{SiC}$ material these substrates have two different faces. The $4 \mathrm{H}(6 \mathrm{H})-\mathrm{SiC}(0001)$ is called the "Si face", and the $4 \mathrm{H}(6 \mathrm{H})-\mathrm{SiC}(000-1)$ is called the " $\mathrm{C}$ face". This standard denomination refers to ideal (bulk truncated) surface. The actual chemical composition depends on the reconstruction of the surface (for brief reviews of the reconstructions of these surfaces see [32-34]). It turns out that genuine reconstructions of the $\mathrm{SiC}$ surface may survive below the first few graphene layers, and thus are relevant for interface properties. These are the $6 \sqrt{ } 3 \times 6 \sqrt{ } 3 \mathfrak{R}\left(30^{\circ}\right)$ reconstruction $-6 \mathrm{R} 3$ in short notationfor the Si face [16, 17, 35, 36], and the $(3 \times 3)$ and $(2 \times 2)_{C}$ reconstructions for the $C$ face $[18,36-39]$. The $6 \mathrm{R} 3$ of the Si face has long been recognised as consisting in a graphitic layer on top of the SiC substrate $[16,17,40]$, but it is only recently (due to the renewal of interest for this system in connection with epitaxial graphene) that the nature of the interface bonding has been investigated (see section 2 for discussion). The structure of the ( $3 \times 3)$ reconstructions of the $\mathrm{C}$ face remains largely unknown although a model has been proposed [41]. The structure of the $(2 \times 2)_{C}$ reconstruction of the $C$ face has been established by quantitative low energy electron diffraction (LEED) analysis [42]. It will be presented in section 3. Since the structure of the interface between the first graphene layer and the substrate is markedly different for the $\mathrm{Si}$ and the $\mathrm{C}$ face of the substrate, we prefer to present the results in two different sections.

The formation of graphene layers starting from genuine $\mathrm{SiC}$ surface reconstructions is obtained by high temperature annealing. The whole process $-\mathrm{SiC}$ surface preparation and 
graphitization- is usually performed under ultra-high vacuum (UHV) conditions for STM investigations, a common practice in surface science. It has been shown however that preparation under UHV results in a rather poor morphology of the graphene layers [19, 35, 36, 38, 43-50]. The films are not homogeneous since patches with a different number of graphene layers coexist on the surface. Moreover the typical size of the (homogeneous) terraces is small, typically $100 \mathrm{~nm}$ or less. Although this is not an issue for STM studies -and can even be advantageous since several phases can be investigated with the same tip-, the heterogeneity may be detrimental for transport properties or for investigations using non local techniques. Non UHV growth techniques have been shown to give samples with much more uniform morphology [15, 48, 49, 51, 52]. However, when surface science techniques such as LEED or photoemission have been used for non-UHV monolayer graphene samples grown on the Si face [48, 51], no obvious difference with UHV grown samples were found. This indicates that the atomic and electronic structure of the interface is similar for both preparation techniques for the Si face, and that STM investigations on UHV grown samples are therefore relevant for non-UHV ones.

The situation may possibly be different for the $\mathrm{C}$ face of the substrate. Most of the pioneering experiments by the group of Walt de Heer $[6,9,12,28]$ have been made on samples grown in nonUHV conditions on this $C$ face. The samples were typically several layers thick [49] which has hampered a direct investigation of the interface by STM and other surface sensitive techniques. The STM data on the interface structure -and the related ab initio calculations- that we present in section 3 of this review have been obtained on samples prepared in UHV, using a significantly lower growth temperature. Whether the characteristics of the interface of UHV grown samples -which are largely determined by the reconstructions of the bare substrate surface- are preserved using non-UHV preparation techniques remains to be established.

\subsection{Experimental techniques}

The main experimental technique considered here is STM. This technique is now well established. It has been reviewed in a number of textbooks [53] and will not be described in this article, which focus on the properties of the material. Ab initio calculations are usually performed within the density functional theory (DFT), for instance the VASP code [54]. For technical details (supercell geometry, choice of the functional and of the pseudo-potential) the reader can refer to the original papers [21, 66-69, 94, 95].

\section{Graphene on the $\mathrm{Si}$ face of $\mathrm{SiC}$ substrates}

As mentioned in section 1.3, the graphitization process leads to heterogeneous samples which contain FLG regions of different thicknesses. Moreover, there is no unambiguous and easy way to know "a priori" how many graphene planes are present on the average on a sample prepared "in-situ" 
(the most readily available surface characterization techniques such as LEED [35, 36] and Auger spectroscopy [49] are only helpful after a calibration). Thus we need a criterion to identify monolayer graphene areas in situ by STM. This is the subject of paragraph 2.1. The structure of the interface derived from ab initio calculations, including a recent model for the 6R3 (or buffer layer) phase, will be presented in section 2.2. For convenience the results of the band structure calculations are also included in section 2.2. In section 2.3 we shall discuss the electronic structure of monolayer and bilayer graphene on the Si face from the point of view of STM experiments. In particular, it will be shown that one specific -and important- feature of the electronic structure of graphene, namely the electronic chirality, can be probed by STM.

\subsection{Identification of monolayer $(M L)$ graphene in STM images}

2.1.1. Atomic contrast. In principle the identification of ML graphene by STM is straightforward. As shown in section 1, the low energy LDOS is similar on A and B sublattices and thus STM images at small sample bias V should reveal a honeycomb pattern. This is exactly what we observe in figure 2-a, and thus we identify this sample area as ML graphene on the Si face [55].

As discussed in section 1.2, the LDOS on the A and B sublattices of the top carbon layer is different for Bernal stacked bilayer graphene (BL). This should lead to a "triangular" contrast in low bias STM images, where every second $\mathrm{C}$ atom (the one of A type) seems to be missing. This corresponds exactly to the observation made on a region identified as BL graphene on the Si face, as shown in figure 2-b [55].

Although the identification of the phases by means of low bias STM images seems deceivingly simple, some points of caution need to be considered. They are listed below:

i) The triangular ( $\mathrm{AB}$ asymmetric) contrast is not specific to the bilayer: Bernal stacked multilayers [56] and finally graphite [57] qualitatively have the same contrast at low bias.

ii) Conversely, non Bernal stacked multilayers (including bilayers) can show a honeycomb contrast very similar to ML graphene. This has been ascribed to a translation or shift $[56,58]$, a rotation [59] or a decoupling [60] between the last two carbon planes.

iii) Even in a regular situation (low bias images, Bernal stacked bilayer) the contrast on BL has been shown to depend on the bias [61] : using moderate positive sample bias $(\mathrm{V} \approx+0.5 \mathrm{~V})$ one may observe a honeycomb pattern. This is due to the fact -quoted in section 1- that the LDOS on A and B sublattices of the top plane become equal a fraction of $\mathrm{eV}$ above $\mathrm{E}_{\mathrm{D}}$.

From these considerations, it would seem useful to get some auxiliary means to identify faithfully the monolayer phase (at least) using STM. The interface contribution to the images can be used for this purpose, and it also allows a rapid identification of ML and BL regions in large scale images [55]. This point is developed in paragraph 2.1.2. We mention here an alternative technique, 
which consists in counting the number of nodes -due to electron scattering at buried interfaces- in the field effect resonances which appear at high bias $(\mathrm{V}>5 \mathrm{eV})$ in $\mathrm{Z}(\mathrm{V})$ spectroscopy [62]

For completeness let us say a few words about the atomic contrast observed in other realizations of monolayer graphene. Exfoliated graphene on silicon dioxide exhibits the honeycomb contrast expected for the ideal material [63]. For graphene on metals the situation is more intricate since the local interaction of the $\mathrm{C}$ atoms with the substrate influences the contrast in the STM images [10]. For instance, in the strongly interacting case of graphene on $\mathrm{Ru}(0001)$, the atomic contrast of $\mathrm{ML}$ graphene changes from honeycomb to triangular within $1 \mathrm{~nm}$, as a consequence of the local registry and interaction- with the surface metal atoms [10].

\subsubsection{Long range (6x6) modulations. The images in figure 2-a show more than the graphene} honeycomb contrast. One can see a long range hexagonal superstructure with period $\approx 1.9 \mathrm{~nm}$ which modulates the apparent height of the plane [55]. This modulation comes from the underlying 6R3 surface reconstruction of the substrate surface (which is thus the interface plane). The 6R3 substrate reconstruction commonly looks like a (6x6) (instead of a 6R3) superstructure of the $\mathrm{SiC}$ surface in most high bias STM images [20, 35, 40, 55, 56, 64, 65]. The expected period of the (6x6) modulation is $1.85 \mathrm{~nm}$ which corresponds quite well to the value found in figure 2-a. Thus the superstructure observed for the first graphene layer is generated by the underlying (6R3) interface. This statement is confirmed by the ab initio calculations presented in section 2.2.2. This super period also shows up, although with reduced amplitude, for the bilayer (figure 2-b) [55]. Indeed, it has been proposed that the measurement of the roughness induced by the interface in the top layer could be used as a fingerprint of the thickness of the FLG sample [56].

As mentioned previously, the 6R3 substrate reconstruction of the Si face involves a graphitic plane on top of the substrate $[16,17,40]$. However no graphene or graphite signal -similar to the one of figure 2- has ever been reported on this surface by STM, even in low bias/high resolution images $[35,55,56,64,65]$. This discrepancy will be explained in the next paragraph where we present the current model for this reconstruction, as derived from ab initio calculations.

\subsection{Atomic and electronic structure of the interface from ab initio calculations}

\subsection{1. principles of ab initio calculations of the interface structure. Due to the large size of the unit} cell, ab initio computations on the 6R3 are quite challenging. However, such results have been reported by two groups $[66,67]$. The initial configuration was a flat graphene layer laid above an unreconstructed (ideal) $\mathrm{SiC}$ surface. After relaxation the $\mathrm{C}$ honeycomb lattice is distorted by an interaction with the substrate, see figure 3-a. In the following, we detail the data of Ref. [67], although essentially similar results were found for the atomic structure of the interface in [66]. 
Calculations were made for one and two $\mathrm{C}$ layers on top of $\mathrm{SiC}$ in this cell. In the first (second) case, the slab contained four bilayers of $\mathrm{SiC}$ (two) and then 1310 atoms (1216). Only the $\Gamma$ point was used. Although in the second case only a small number of substrate bilayers was used, it was checked that this had no effect on the relaxation of the first $\mathrm{C}$ layer.

\subsubsection{The first C layer on SiC : the buffer layer (or nanomesh). Images of the total charge density} after relaxation of the system are shown in figure 3-b and 3-c. The first $\mathrm{C}$ layer is strongly distorted by the formation of covalent bond with the substrate in the region where the graphene and $\mathrm{SiC}$ lattices are in registry. This inhomogeneous bonding results in a complex mosaic pattern and in an apparent 6x6 periodicity of the $\mathrm{C}$ layer (red diamond of Fig. 3-b) while the actual periodicity remains the $6 \mathrm{R} 3$ cell (blue diamond in Fig. 3-b) used for the calculation. Covalent bonds are formed between the Si atoms of the last $\mathrm{SiC}$ layer and the $\mathrm{C}$ atoms of the $\mathrm{C}$ layer, as shown in the cross-section view of the total charge density of figure 3-c. This prevents any graphitic electronic properties for this layer. Indeed, the computed band structure does not exhibit the linear dispersion of the $\pi$ band characteristic of graphene [66] confirming preliminary calculations $[68,69]$ in a simplified geometry. In agreement with these theoretical findings, ARPES data have shown that the "Dirac cones" typical for the band structure of graphene were absent on the $6 \mathrm{R} 3$ phase but that the $\sigma$ bands were fully developed [37]. $\mathrm{C}$ atoms bonded to Si are closer to the substrate as shown in figure 3-c, which leads to a strong corrugation $(0.12 \mathrm{~nm})$ of the surface layer [67]. We called this first $\mathrm{C}$ layer (6R3 phase) a buffer layer since it decouples the following $\mathrm{C}$ layers from the substrate and it allows them to behave like graphene mono or multilayers as shown below. Recent investigations by transmission electron microscopy [70, 71] have confirmed the existence of this buffer layer strongly bonded to the Si surface atoms of the SiC substrate.

\subsection{3. ab initio calculations for two and three C layer: monolayer and bilayer graphene. The graphene} nature of the film is recovered for the second $\mathrm{C}$ layer. This second $\mathrm{C}$ plane follows the morphology of the buffer layer - like a carpet- and as a consequence presents a honeycomb atomic lattice with a superimposed 6x6 periodicity (Fig. 4-a and 4-b) [66, 67]. This is consistent with the aforementioned STM experiments. The band structure computed for this structure shows the $\pi$ bands characteristic of graphene with a Fermi level that lies around $0.5 \mathrm{eV}$ above the Dirac point $[21,66]$. Again these results confirm the preliminary calculations performed in a simplified geometry $[68,69]$. The second $C$ plane is thus actually the first graphene layer. It is electron doped by a charge transfer from the substrate in agreement with ARPES experiments [13, 14]. Going further into details, the band structure calculations in the full 6R3 structure [66] reveal the opening of a small gap (250 meV wide) at the Dirac point, in agreement with one set of ARPES data [14]. The gap has been ascribed to a residual coupling between the graphene plane $\left(2^{\text {nd }} \mathrm{C}\right.$ layer $)$ and the buffer layer. Conversely, computations in 
the simplified geometry -as well as our own computations in the full 6R3 structure- do not show this gap [21, 68, 69], in agreement with another set of ARPES data [13]. It is not the purpose of this paper to comment further on this controversy. Anyway, the complex geometry of the first carbon layer (buffer layer) generates soft ripples (with amplitude $0.04 \mathrm{~nm}$ ) in the honeycomb lattice of the graphene - second C- layer. The calculated corrugations and morphologies of the two first $\mathrm{C}$ layers are in very good agreement with the STM images [67]. On this Si face, the interaction with the substrate thus disturbs at least the two first $\mathrm{C}$ layers. On the other hand, all experiments demonstrate that the graphene is epitaxial on this face. The long range orientation of the graphene planes is then imposed by the substrate.

Calculations of a third C layers on the Si face have been performed in the simplified geometry only [68]. The Bernal -graphite-like- stacking of $\mathrm{C}$ planes was considered since it was found from experiments on the Si-face. The results are in good agreement with ARPES spectra [72] and show the opening of a gap at the Dirac point. The three $\mathrm{C}$ layers behave as a buffer layer plus a graphene bilayer. The gap comes from the asymmetry of the bilayer and is due to a different doping level of the two layers: indeed charge transfer is larger for layers closer to the interface [73] for as-grown films. The gap closes when the two carbon layers are made equivalent by external doping [72].

2.2.4. Beyond the buffer layer model, STM experiments. One feature of STM imaging of graphene on $\mathrm{SiC}$ is that the buried interface can be directly observed at high bias [20,64], although with a reduced contrast compared to the bare 6R3 reconstruction. This "transparency" at high bias has been ascribed to the energy dependence of the density of states [65] -with a large interface DOS at high energy- or to the short attenuation length perpendicular to the surface of the $\pi$ states of graphene (due to the high value of the parallel wave vector close to the $\mathrm{K} / \mathrm{K}^{\prime}$ points) [20,55]. Anyway, this feature gives the opportunity to investigate the structure of the interface plane below the graphene layer, which, according to the results presented in section 2.2.3 should be the buffer layer. It has generally been found $[35,55,56,65,74]$ that this interface plane was somewhat disordered, as shown in figure 5. Moreover, unexpected features -with respect to the buffer layer model of section 2.2.2- show up in the high bias images (see e. g. the arrow in figure 5). A series of variable bias STM images reported by Rutter et al. indicates that such structures look like pyramids or tetramers, and that they are located below the raised regions of the graphene surface layer [65]. It thus appears that the actual interface structure is significantly more complex -and disordered- than the theoretical model shown in section 2.2.2. However, as quoted above, this model captures the essential characteristics of the interface. Additionally, the STM images of figure 5 show that interface electronic states give a significant -if not dominant [65]- contribution to the tunnelling current in the bias range where the Dirac point is expected, this is around $-450 \mathrm{mV}$. This remark is important to understand the results of scanning tunnelling spectroscopy (STS) discussed below (section 2.3.1). 


\subsection{Electronic structure of monolayer and bilayer graphene from STM experiments}

The theoretical band structure computed for monolayer and bilayer graphene, as well as the corresponding ARPES data have been presented in section 2.2. In this section we consider results which are more specific to the STM technique. We first briefly discuss tunnelling spectroscopy data in section 2.3.1, and then we describe the analysis of the standing wave patterns generated by defects on graphene. We show that the chiral nature of the electronic states is reflected in theses quantum interference patterns.

2.3.1. Scanning tunneling spectroscopy. As discussed in section 2.2.2, both theoretical calculations and photoemission data demonstrate that the band structure of the first graphene layer $\left(2^{\text {nd }} \mathrm{C}\right.$ plane) closely resembles the one of free standing graphene close to the Dirac point. Thus we could naïvely expect that STS experiments on the first graphene layer would exhibit V shape spectra at low energy, with a minimum located at the Dirac point, this is approximately $450 \mathrm{meV}$ below $\mathrm{E}_{\mathrm{F}}$. It turns out that this is definitely not the case. Features related to the Dirac point are hardly observed in the spectra taken on monolayer graphene [56, 65, 74], except for the data reported in [75]. The absence of a clear signature of the Dirac point in STS for monolayer graphene is thought to be due to the large contribution of the interface states to the tunneling current around $-450 \mathrm{mV}$ [56, 65], as quoted above (section 2.2.3). For multilayer graphene, the interface contribution to the total current decreases and the data show minima at biases where the Dirac point is expected from ARPES data [56].

\subsubsection{Quantum interferences patterns, wavefunction symmetry and electronic chirality. In a two} dimensional (2D) system such as graphene, disorder has a strong impact on the electronic properties, because of the high efficiency of scattering phenomena. Quasiparticle elastic scattering generates quantum interferences (QIs), i.e. periodic modulations of the LDOS, which are known to affect the macroscopic electronic-transport properties of the system. Such QIs, which are related to the well known Friedel oscillations in 2D metals, can be probed at the nanometer scale using an STM [76]. This can be achieved in the standard topographic mode by recording constant current images at low sample bias, or in the spectroscopic mode by recording $\mathrm{dI} / \mathrm{dV}$ images at higher bias $\mathrm{V}$. The former (latter) mode roughly corresponds to LDOS maps at $\mathrm{E}_{\mathrm{F}}\left(\mathrm{at} \mathrm{E}_{\mathrm{F}}+\mathrm{eV}\right)$. Both modes have been widely used in the past for analysing QIs on noble metal surfaces or on metallic 2D systems on semiconductor substrates [76-82].

In a simple picture, any point defect embedded in a 2D system allows elastic scattering between two states $\mathbf{k}_{\mathbf{F}}$ and $\mathbf{k}_{\mathbf{F}}$ ' of the $2 \mathrm{D}$ Fermi surface (FS). This leads to QIs with wavevector $\mathbf{k}_{\mathbf{F}}$ '- $\mathbf{k}_{\mathbf{F}}$, with a weight depending on the topology of the FS. For a standard 2D system with a single circular FS, LDOS modulations at $\mathrm{E}_{\mathrm{F}}$ are dominated by backscattering processes, i.e. coupling between opposite states $+\mathbf{k}_{\mathbf{F}}$ and $-\mathbf{k}_{\mathbf{F}}$. The corresponding period of the modulations is $\pi / \mathrm{k}_{\mathrm{F}}$, which is for 
instance the value reported from low bias constant current images on noble metal surfaces [76, 81]. Following the same argument, the backscattering processes between states $+\mathbf{k}$ and $-\mathbf{k}$ of any energy $\mathrm{E}$ lead to LDOS modulations of period $\pi / \mathrm{k}$, which can be directly measured on conductance maps at sample bias $\mathrm{eV}=\mathrm{E}$. This gives a unique opportunity to probe the dispersion relation $\mathrm{E}(\mathrm{k})$ of the $2 \mathrm{D}$ system $[76,78,82]$.

In the following, we present the analysis of the QIs measured by STM on monolayer and bilayer graphene on $\mathrm{SiC}(0001)$. The main purpose is to extract from such analysis a clear picture of the scattering mechanisms occurring in these systems, and also an evaluation at the nanometer scale of fundamental quantities such as the Fermi wave vector (and thus the electronic doping), the dispersion relation, and also the electronic chirality.

As a starting point, let us mention that monolayer and bilayer graphene on $\operatorname{SiC}(0001)$ show a very similar FS, despite a markedly different electronic dispersion as discussed in paragraph 1 . The common schematic FS for both systems is depicted in Fig. 6-a. It is derived from ARPES measurements, performed separately on monolayer and bilayer graphene on $\operatorname{SiC}(0001)[13,72]$. The FS consists of two tiny circular pockets of radius $\mathrm{q}_{\mathrm{F}} \approx 0.6 \mathrm{~nm}^{-1}$, surrounding the $\mathrm{K}$ and $\mathrm{K}$ ' points of the first Brillouin zone. Depending on the disorder present in the system (sharp impurities, long range ripples, curving of the graphene layer at substrate steps, localized interface states...), we expect from the shape of the FS two different scattering mechanisms: Intervalley scattering, which couple states of the two non-equivalent pockets of the FS (as illustrated on Fig. 6-b), and intravalley backscattering, which couple opposite states of the same FS pocket (as shown on the left picture on Fig. 6-d).

The STM image displayed in Fig. 6-c demonstrates that QIs associated to intervalley scattering processes are indeed observed on monolayer graphene on $\mathrm{SiC}(0001)$ [55]. The image, recorded at $+0.1 \mathrm{~V}$, includes one sharp defect on the left. As previously explained, the image is strongly affected by the interfacial $6 \times 6$ modulation, and by possible interface states. In addition, very clear fringes are found surrounding the defect, with a lateral extension of a few nms. The fringes are tilted by an angle of $30^{\circ}$ with respect to the graphene lattice directions (which show up at the right bottom corner of the image), and are separated by a short period of $\sim 3.7 \AA$. This value is close to $2 \pi / \Gamma \mathrm{K}$, in agreement with the intervalley scattering process depicted in Fig. 6-b. Because of the symmetry of the FS, the fringes pattern forms a $(\sqrt{3} \times \sqrt{3}) \mathrm{R}\left(30^{\circ}\right)$ superstructure with respect to the (1×1) graphene lattice. This superstructure has also been reported on bilayer graphene on $\mathrm{SiC}(0001)$ $[55,83]$ and is commonly observed on HOPG surfaces [84]. Actually, any atomic-sharp impurity in contact with (or included in) a graphitic atomic layer is likely to induce this superstructure, because of its associated short-range impurity potential which can couple states of different ( $\mathrm{K}$ and $\mathrm{K}$ ') valleys of the FS. The STM observation of areas with $(\sqrt{3} \times \sqrt{3}) \mathrm{R}\left(30^{\circ}\right)$ superstructures is a clear evidence that such scatterers are present in our system, either on monolayer or bilayer terraces (most of these defects are probably generated by the graphitisation process itself). We also conclude from such observations 
that the electronic structure at the surface is consistent with the FS shown in Fig. 6-a, built on electronic states with wave vector close to K and K' points.

We now focus on the possible intravalley backscattering processes, which can be generated by any (long or short range) potential associated to disorder. From the FS depicted on Fig. 6-a, the corresponding QIs should have a wavevector $2 \mathrm{q}_{\mathrm{F}}$, and thus a period $\pi / \mathrm{q}_{\mathrm{F}} \approx 5.2 \mathrm{~nm}$. This is more than an order of magnitude higher than the period of the QIs associated to intervalley scattering. Accordingly, high resolution STM images of large homogeneous terraces have to be achieved in order to capture such large period QIs. This is the case on Fig. 6-d, showing a $50 \times 50 \mathrm{~nm}^{2}$ constant current image with two neighbouring bilayer and monolayer graphene terraces (respectively on the left and on the right) [85]. The image was recorded in the group of Prof. K. Kern in MPI Stuttgart. It was acquired at $4 \mathrm{~K}$ and very low sample bias $(+1 \mathrm{mV})$, and thus reflects the surface LDOS at the Fermi level with negligible energy broadening. A clear long-range modulation, of wavelength $5.2 \pm 0.3 \mathrm{~nm}$, is found on the the graphene bilayer terrace (left terrace on image 7-d). Such modulation has also been reported by Rutter et al. [83], and it is attributed to the QIs with wavevecor $2 \mathrm{q}_{\mathrm{F}}$ associated to intravalley backscattering (as illustrated in the left boxed area of Fig. 6-d). Surprisingly, we do not find such LDOS modulation on monolayer graphene (right terrace of Fig. 6-d), which is puzzling if we keep in mind that the two systems have almost the same FS.

The absence of $2 q_{F}$ LDOS modulation on monolayer graphene is a consequence of the electronic chirality -or pseudo spin- quoted in section 1 . The honeycomb structure of graphene leads to a specific symmetry of its low energy states. As a consequence, intravalley backscattering -i. e. scattering between states with wavevectors $\mathbf{K}+\mathbf{q}_{\mathbf{F}}$ and $\mathbf{K}-\mathbf{q}_{\mathbf{F}}$ (or equivalently $\mathbf{K}^{\prime}+\mathbf{q}_{\mathbf{F}}$ and $\mathbf{K}^{\prime}-\mathbf{q}_{\mathbf{F}}$ )- can not occur for slowly varying potentials [24], nor for short range potentials with the AB sublattice symmetry [88]. It was recently shown that for short range potentials breaking the sublattice $A B$ symmetry, intravalley backscattering is restored. However, the related $2 \mathrm{q}_{\mathrm{F}} \mathrm{LDOS}$ modulations are phase shifted by $\pi$ between the A and B sublattices [86]. Thus the two contributions cancel each other when averaged on the lattice unit cell, drastically reducing the (coarse grained) amplitude of the $2 \mathrm{q}_{\mathrm{F}}$ LDOS modulations, which display a $1 / \mathrm{r}^{2}$ decay instead of the characteristic $1 / \mathrm{r}$ decay found in conventional 2D systems [87-89]. As a consequence, the Fourier Transform (FT) of low bias images of monolayer graphene -in presence of point defects- does not display a ring with radius $2 \mathrm{q}_{\mathrm{F}}$ at the origin $[85,87,88]$. This behaviour results from the electronic chirality (pseudo spin) of monolayer graphene. Although we do not precisely know if part of the atomic-size defects in our system do locally break the AB symmetry, we can conclude from the non-detection of QIs of period $5.2 \mathrm{~nm}$ that the electronic chirality of monolayer graphene on $\mathrm{SiC}(0001)$ is the one predicted for free standing graphene. The observation of such QIs on bilayer graphene (left terrace of Fig. 6-d) is also in perfect agreement with theory. Indeed, as mentioned in paragraph 1, the symmetry (electronic chirality) of the low energy states in Bernal stacked bilayer graphene enables the intravalley backscattering, and the 
associated QIs decay following the standard $1 / \mathrm{r}$ law [87]. Hence a ring with radius $2 \mathrm{q}_{\mathrm{F}}$ shows up at the origin in the FT of low bias images of bilayer graphene in presence of point defects $[83,85,87]$. This is similar to the situation for normal 2D electron gases [81], but quite different from the case of monolayer graphene.

To conclude this section, we make two remarks. Firstly, (incomplete) circles with radius $2 \mathrm{q}_{\mathrm{F}}$ actually show up in the FT of low bias images surrounding the $\mathrm{K}\left(\mathrm{K}^{\prime}\right)$ points in reciprocal space for both monolayer and bilayer graphene, their shape being in agreement with the theory [85, 87]. The value of the Fermi wavevector obtained from these measurements fits with ARPES data. Secondly, the FT of conductance maps recorded at different biases allows for an estimate of the dispersion of the $\pi$ bands in the vicinity of the Fermi level, for both bilayer [83, 90] and monolayer graphene [83], as for usual 2D electron gases.

\section{Graphene on the $\mathrm{C}$ face of $\mathrm{SiC}$ substrates}

It has long been known that the graphitization processes of $\mathrm{SiC}$ substrates in UHV are different on the $\mathrm{C}$ face and on the $\mathrm{Si}$ face $[16,18]$. Graphitization is faster on the $\mathrm{C}$ face, and more importantly the graphitic layers show a significant amount of rotational disorder, which results in "streaks" or "rings" in the LEED patterns $[16,18]$. Some preferential orientations of the graphitic layers are nevertheless found in the LEED patterns, but they apparently depend on the preparation procedure $[36-38,49,52,91]$. In the following we shall focus on the first graphene layer grown on the C face. Until recently the growth of one layer could be controlled only in UHV conditions, but recent reports show that it can also be produced in non UHV environments [92, 93]. To our knowledge no detailed investigation of the structure of the interface has been made yet by STM on such non UHV samples.

Section 3 is organized as follows. In the first paragraph we present STM data on UHV prepared samples which demonstrates, in agreement with other techniques such as photoemission, that the first graphene layer grows directly on genuine reconstructions of the $\mathrm{SiC}$ surfaces, thus without any buffer layer. Two different interfaces are found, corresponding to two different SiC surface reconstructions. In the second paragraph a brief account of the structure of one kind of interface is given, and in the third paragraph ab initio calculations on the second kind of interface will be presented. We do not discuss the case of multilayers samples in this paper which is devoted to interface studies. Let us only mention that the stacking of successive $\mathrm{C}$ planes is generally not of the Bernal (graphite) type, at variance with the situation for the Si face. Adjacent planes are frequently rotated [49, 59], which results in an "electronic decoupling" of the layers [52]. This decoupling explains why the physical properties typical for ML graphene have been observed $[6,8,12]$ even for FLG samples grown on the $\mathrm{C}$ face of $\mathrm{SiC}$ substrates. 


\subsection{Structure of the interface}

A typical LEED pattern of a slightly graphitized sample (less than one graphene layer) is shown in figure 7-a. It displays the characteristic features of UHV prepared samples [18, 36-39, 91]. The circles indicate the $(1 \times 1)$ spots of the substrate. The other spots are related to the $(3 \times 3)$ and $(2 \times 2)_{C}$ surface reconstructions of the $\mathrm{SiC}$ surface, with arrows pointing to the faint $(2 \times 2)_{\mathrm{C}}$ structures. The "streaks" or "arcs" indicated by the dashed (quarter) circle are due to the graphene layer. Obviously, although preferential orientations exist, the orientation of the first graphene layer with respect to the surface is not unique. This suggests some "rotational disorder", which persists for multilayer samples as reported by several groups $[9,15,18,37,52,59]$.

A large scale STM image of the same sample, taken on a single terrace of the substrate surface, is displayed in figure 7-b [38]. Atomically resolved images [45] reveal that the lowest (darkest) level marked " $3 \times 3$ " is the bare $(3 \times 3)$ reconstructed substrate surface, as shown by comparison with previously published data $[32,41]$. FLG denotes multilayer islands. Most of the area is covered with two types of islands, hereafter called G_2x2 and G_3x3, which consists of monolayer graphene islands on the $(2 \times 2)_{\mathrm{C}}$ and $(3 \times 3)$ reconstructions of the $\mathrm{SiC}$ surface respectively, as shown below. Their typical lateral size is about ten nanometres. Their apparent height with respect to the bare (3x3) surface is $2.6 \AA\left(G_{-} 2 \times 2\right)$ and $3.1 \AA\left(G_{-} 3 \times 3\right)$ in figure 7-b, which is indeed consistent with one graphene layer.

Figure 8 displays images taken at a boundary between a $G \_2 \times 2$ (on the right side of figure 8 -a) and a $\mathrm{G}_{-} 3 \times 3$ island (on the left side of figure 8-a) [38]. Zoomed-in variable bias images were taken in boxed areas of figure 8-a. Due to the "transparency" of the graphene mentioned in section 2.2.4, high bias images are used to reveal the structure of the underlying interface. Both negative (figures 8-b and 8-c) and positive (not shown) high bias images on the G_3 3 3 and G_2x2 islands are similar to the one reported for the bare $(3 \times 3)[32,41,45]$ and $(2 \times 2)_{C}[32,42]$ reconstructions of the substrate respectively. Low bias images (figures 8-d and 8-e) exhibit an ordered lattice with the periodicity of graphene (and at least locally with a honeycomb contrast, see insets). This establishes the structure of the islands quoted above. Essentially similar STM observations have been reported in [36].

Low bias STM images (figure 8-d and 8-e) demonstrate that the $\mathrm{p}_{\mathrm{z}}$ states of graphene are present close to the Fermi level on both $\mathrm{G} \_3 \times 3$ and $G \_2 \times 2$ islands. In detail, the honeycomb contrast of graphene appears almost unperturbed on the $\mathrm{G} \_3 \times 3$ islands whatever the orientation of the $\mathrm{C}$ layer with respect to the substrate $[38,39,45]$. This points to a very weak electronic coupling between the graphene plane and the underlying $\operatorname{SiC}(3 \times 3)$ surface. The graphene layer seems to be slightly more influenced by the underlying $\operatorname{SiC}(2 \times 2)_{C}$ reconstruction on $\mathrm{G}_{-} 2 \times 2$ islands since triangular structures (arrows in figure 8-e) arranged with a (2x2) periodicity -which look like "missing atoms"1- show up at

\footnotetext{
${ }^{1}$ It is likely that these atoms are not really missing: they appear darker due to a LDOS effect, see ab initio calculations in section 3.3 where these structures are called « switch off » atoms.
} 
the boundary of patches where the honeycomb contrast is observed. This indicates a non negligible local interaction between graphene and the underlying $(2 \times 2)_{C}$ reconstruction [38] (this statement will be made more quantitative by means of ab initio calculations in section 3.3). However, the graphene contrast remains visible in the patches of the G_2x2 islands (Fig. 8-e), and the observation of structures with the atomic spacing of graphite even in the perturbed areas demonstrates that this interaction is not strong enough to remove all the $\mathrm{C} \mathrm{p}_{\mathrm{z}}$ states from the vicinity of the Fermi level. These observations are at variance with the STM experiments made on the Si face of SiC quoted in section 2: in this later case the coupling between the first graphitic layer (buffer layer) and the substrate is strong. The graphene $\mathrm{C} \mathrm{p}_{z}$ states (or $\pi$ bands) are removed from the vicinity of the Fermi level (the Dirac cones are destroyed) and subsequently no graphitic or honeycomb contrast is observed in low bias images. STM experiments thus indicate a rather weak coupling at the interface between the first graphene layer and the $\mathrm{SiC}$ substrate for the $\mathrm{C}$ face, especially for the $\mathrm{G} \_3 \times 3$ islands.

The conclusions derived from STM data are supported by independent observations, which however do not discriminate between $\mathrm{G} \_2 \times 2$ and $G \_3 \times 3$ islands. The "rotational disorder" seen in LEED patterns suggests that the graphene-substrate interaction is not strong enough to impose a definite orientation to the $\mathrm{C}$ layer [36-38]. Core level photoemission data do not provide any evidence for a covalently bound $\mathrm{C}$ layer at the graphene-substrate interface, at variance with the case of the $\mathrm{Si}$ face $[36,37]$. Moreover, ARPES data reveal that the $\pi$ bands of graphene (Dirac cones) are formed even for submonolayer graphene coverage, indicating a weak graphene-substrate coupling [37]. The picture which emerges from this set of data is that, at least for UHV grown samples, the first graphene layer is weakly perturbed by the underlying reconstructed $\mathrm{SiC}$ surface.

\subsection{The $G \_3 x 3$ interface}

In figure 8-a a superstructure with period $4.1 \mathrm{~nm}$ (indicated by the double-sided arrow) shows up on the $\mathrm{G}_{-} 3 \times 3$ island. Different superstructures with periods in the $\mathrm{nm}$ range were observed on a collection of G_3x3 islands $[39,45]$. They correspond to different orientations of the graphene layer with respect to the underlying $\mathrm{SiC}$ substrate, and a detailed analysis has shown that these superstructures were actually Moire patterns (MP's) [39]. The MP's were attributed to small undulations -typically $0.02 \mathrm{~nm}$ peak to peak- of the graphene layer from bias dependent measurements. Thus the corrugation of the graphene layer on the $\mathrm{SiC}(3 \times 3)$ reconstruction of the $\mathrm{C}$ face is comparable to (and even smaller than) the undulations of the first graphene layer (second $\mathrm{C}$ plane) on the Si face, and should thus bring minimal perturbation to the electronic structure of the material [39].

A G_3 3 3 island with graphene layers rotated by $30^{\circ}$ (with respect to the $\mathrm{SiC}$ substrate) is shown in figure 9. The MP observed on this island corresponds to a 6R3 unit cell (dashed line in figure 9) with respect to the $\mathrm{SiC}(1 \mathrm{x} 1)$ surface [39]. The inset shows that the honeycomb contrast of graphene 
is observed at low bias on this island. This is totally different from the situation encountered for the buffer layer -first $\mathrm{C}$ plane- on the $\mathrm{Si}$ face (also rotated by $30^{\circ}$ ) where an apparent (6x6) supercell is observed and where no graphene signal can be detected in low bias STM images. This is also at variance with ab initio computations performed for a graphene layer rotated by $30^{\circ}$ and interacting with the unreconstructed (ideal) $\mathrm{C}$ face of $\mathrm{SiC}$ [67-69]. In these calculations, the stable structure was close to a buffer layer phase: strong covalent bonds form between the $\mathrm{SiC}$ surface atoms and the graphene layer, and subsequently no $\pi$ states are found in the vicinity of the Fermi level. This would prevent the observation of a honeycomb contrast at low bias. This comparison indicates that the $(3 \times 3)$ reconstruction of the $\mathrm{C}$ face of $\mathrm{SiC}$ efficiently passivates the substrate surface, leading to a weak coupling with the graphene layer [39].

Spectroscopic investigations were performed in Ref. [39] (not shown). They indicate that the bare (3x3) surface exhibit a low DOS in an energy window extending from -1.4 to $+0.1 \mathrm{eV}$ (and thus a surface gap or pseudogap since a residual density of in-gap states is observed). The Fermi level of graphene is located just below the top of this gap, therefore there are very few substrate states to which the $\pi$ bands of graphene can couple in a large energy range $(>1 \mathrm{eV})$ below the Fermi level. These findings are in general agreement with photoemission data for the bare and lightly graphitized $\operatorname{SiC}(3 \times 3)$ surfaces [37].

\subsection{The $G \_2 x 2$ interface}

A structural model has been proposed previously for the $\mathrm{SiC}(2 \times 2)_{C}$ reconstruction [42]. This allows ab initio calculations to be performed in order to investigate in detail the nature of the substrate-graphene interaction on G_2x2 islands. A bulk truncated $(2 \times 2)$ surface presents 4 dangling bonds (DB) per unit cell, one for each $\mathrm{C}$ surface atom. In the model proposed for the reconstructed $(2 \times 2)_{C}$ surface, a Si adatom saturates three $\mathrm{DB}$ and one $\mathrm{C}$ atom (the rest atom) remains unbounded [seubert], see figure 10-a. Furthermore, a charge transfer occurs from the adatom to the restatom so that all DB are either filled or empty [94]. This charge transfer has been verified in STM images of the $\mathrm{SiC}(2 \times 2)_{\mathrm{C}}$ reconstruction observed at high bias through the graphene layer [95]. The SiC surface is thus passivated, and it is semiconducting. Interaction between graphene and $\mathrm{SiC}$ should then be much smaller than on the unreconstructed $\mathrm{Si}$ face (section 2.2.2). In the model calculation of the $\mathrm{G}_{-} 2 \times 2$ interface (figure 10-b), a 5x5 graphene cell is superimposed without rotation to a $4 \times 4 \mathrm{SiC}$ cell with the $(2 \times 2)_{C}$ geometry presented above [94] These two cells are nearly commensurate. The total energy remains nearly unchanged when the graphene - $\mathrm{SiC}$ surface relative position is shifted laterally: the energy variation is smaller than $15 \mathrm{meV}$ for the whole $4 \mathrm{x} 4$ cell [94]. Thus there is no preferred registry of the graphene layer with respect to the substrate. This result is consistent with the existence of a rotational disorder observed experimentally for the first layer (section 3.1). 
Band structure calculations for the fully relaxed interface are shown in figure 10-c [94]. The results for the graphene- $(2 \times 2)_{\mathrm{C}}$ interface (resp. free standing graphene) are displayed as black (resp. red) lines. The band structure of the interface exhibits the characteristic $\pi$ bands of graphene (Dirac cones) in the vicinity of the Dirac point. This demonstrates that the graphene-substrate interaction hardly modifies the low energy electronic structure of graphene on the $\operatorname{SiC}(2 \times 2)_{C}$ surface. Together with the rather large graphene adatom distance $(0.31 \mathrm{~nm})$ found for the fully relaxed structure, this confirms a rather weak graphene-substrate coupling. A small interaction is nevertheless observed between the $\mathrm{SiC}(2 \times 2)_{\mathrm{C}}$ surface and the graphene layer. It gives rise to an anticrossing of the graphene and $\mathrm{Si}$ adatom bands at about $0.75 \mathrm{eV}$ above the Dirac point in the dispersion curve of Fig. 10-c. But at variance with the case of the unreconstructed $\mathrm{Si}$ face, here, the low energy dispersion for the first $\mathrm{C}$ layer is already linear in agreement with ARPES experiments [37].

Theoretical maps of the integrated LDOS computed in the plane just above the surface [94] (not shown) show fair overall agreement with STM images recorded for a G_2x2 island with the same geometry, i. e. for a graphene layer aligned with the $\mathrm{SiC}(2 \times 2)_{\mathrm{C}}$ substrate [95]. In particular, the calculations reveals "switched-off" $\mathrm{C}$ atoms in the graphene layer, which would correspond to the "missing atoms" in the STM images (section 3.1). This "switching-off" is an electronic effect: the 2D electron gas of graphene interacts with the dangling bond of the substrate Si adatom, creating a dip in the graphene LDOS [94].

\section{Summary and conclusions}

The combination of STM and ab initio calculations has allowed a quite accurate description of the atomic and electronic structure of the graphene-SiC interface for the two polar faces ( $\mathrm{Si}$ and $\mathrm{C}$ face) of the substrate. Specifically, on the Si face, the presence of a strongly bound graphene plane (buffer layer) has been established from ab initio calculations, while STM data have revealed that more complex features, leading to a disordered interface, could also be present. The graphene electronic properties (linear dispersion and chirality) are recovered for the second $\mathrm{C}$ plane. The next $\mathrm{C}$ layer, being Bernal stacked as in graphite, does not exhibit the characteristic signatures of graphene. For the $\mathrm{C}$ face the coupling of the first $\mathrm{C}$ plane to the reconstructed surfaces of the substrate is much weaker (although reconstruction dependent), which leads to graphene-like properties at low energy and to a rotational disorder. This weak coupling is most probably due to the persistence of the bare substrate reconstructions below the graphene layer. For both surfaces, these results are largely supported by independent measurements such as photoemission spectroscopy.

\section{Acknowledgments}

This work was supported by the French ANR ("GraphSiC” Project No. ANR-07-BLAN0161), by the Région Rhône-Alpes (“Cible07”, “Cible08” and "Cluster micro-nano" programs) and by 
the "Fondation Nanosciences" (project RTRA "DispoGraph"). F.H. held a doctoral support from la Région Rhône-Alpes. We acknowledge stimulating discussions with I. Brihuega and C. Bena. We thank A. Mahmood for valuable collaboration. 


\section{Rererences}

[1] Wallace P R 1947 Phys. Rev. 71622

[2] Novoselov K S, Geim A K, Morozov S V, Jiang D, Zhang Y, Dubonos S V, Grigorieva I V and Firsov A A 2004 Science 306666

[3] Berger C et al 2004 J. Phys. Chem. B 10819912

[4] Novoselov K S, Geim A K, Morozov S V, Jiang D, Katsnelson M I, Grigorieva I V, Dubonos A A and Firsov A A 2005 Nature 438197

[5] Zhang Y, Tan Y-W, Stormer H L and Kim P 2005 Nature 438201

[6] Berger C et al 2006 Science 3121191

[7] Geim A K and Novoselov K S 2007 Nat. Mater. 6183

Katsnelson M I and Novoselov K S 2007 Solid State Commun. 1433

[8] de Heer W A et al 2007 Solid State Commun. 14392

[9] Castro Neto A H, Guinea F, Peres N M R, Novoselov K S and Geim A K 2009 Review of Modern Physics 81109

[10] Winterlin J and Bocquet M-L Surf. Sci. 20096031841

[11] Keun Soo Kim et al 2009 Nature (London) 145706

Xuesong Li et al 2009 Science 3241312

Reina A et al 2009 Nanoletters 930

Youngbin Lee et al 2010 Nanoletters 10490

[12] Sadowski M L, Martinez G, Potemski M, Berger C and de Heer W A 2006 Phys. Rev. Lett. 97 266405

[13] Bostwick A, Ohta T, Seyller T, Horn K and Rotenberg E 2007 Nature Physics 336

Bostwick A, Ohta T, McChesney J L, Emtsev K V, Seyller T, Horn K and Rotenberg E 2007 New J. Phys. 9385

[14] Zhou S Y, Gweon G H, Fedorov A V, First P N, de Heer W A, Lee D-H, Guinea F, Castro Neto A H and Lanzara A 2007 Nature Materials 6770

[15] Hass J, Feng R, Li T, Li X, Song Z, de Heer W A, First P N, Conrad E H, Jeffrey C A and Berger C 2006 Appl. Phys. Lett. 89143106

[16] van Bommel A J, Crombeen J E and van Tooren A 1975 Surf. Sci. 48463

[17] Forbeaux I, Themlin J-M and Debever J-M 1998 Phys. Rev. B 5816396

[18] Forbeaux I, Themlin J-M and Debever J-M 1999 Surf. Sci. 4429

[19] Tromp R M and Hannon J B 2009 Phys. Rev. Lett. 102106104

[20] Charrier A et al 2002 J. Appl. Phys. 922479

[21] Varchon F 2008 Thesis manuscript available at: http://tel.archives-ouvertes.fr/tel-00371946/fr/ [22] Katsnelson M I, Novoselov K S and Geim A K 2006 Nature Phys. 2, 620 
[23] Bena C and Montambeaux G 2009 New. J. Phys. 11095003

[24] Ando T 2005 J. Phys. Soc. Jpn. 74777

Ando T and Nakanishi T 1998 J. Phys. Soc. Jpn. 671704

[25] Young A F and Kim P 2009 Nature Physics 5222

[26] Stander N, Huard B and Goldhaber-Gordon D 2009 Phys. Rev. Lett. 102026807

[27] McCann E, Kechedzhi K, Fal'ko V I, Suzuura H, Ando T and Altshuler B L 2006 Phys. Rev. Lett. 97146805

[28] Wu X, Li X, Song Z, Berger C and de Heer W A 2007 Phys. Rev. Lett. 98136801

[29] Tikhonenko F V, Kozikov A A, Savchenko A K and Gorbachev RV 2009 Phys. Rev. Lett. 103 226801

[30] Wang Z F, Li Q, Su H, Wang X, Shi Q W, Chen J, Yang J and Hou J G 2007 Phys Rev B 75 085424

[31] McCann E, Abergel D S L and Fal'ko V I 2007 Solid State Commun. 143110

Nilsson J, Castro Neto A H, Guinea F and Peres N M R 2008 Phys. Rev. B 78045405

[32] Bernhardt J, Nerding M, Starke U and Heinz K 1999 Materials Science and Engineering B6162207

[33] Heinz K, Bernhardt J, Schardt J and Starke U 2004 J. Phys.: Condens. Matter 16 S1705

[34] Seyller T 2006 Appl. Phys. A 85371

[35] Riedl C, Starke U, Bernhardt J, Franke M and Heinz K 2007 Phys. Rev. B 76245406

[36] Starke U and Riedl C 2009 J. Phys.: Condens. Matter 21134016

[37] Emtsev K V, Speck F, Seyller T, Ley L and Riley J D 2008 Phys. Rev. B 77155303

[38] Hiebel F, Mallet P, Varchon F, Magaud L and Veuillen J-Y 2008 Phys. Rev. B 78153412

[39] Hiebel F, Mallet P, Magaud L and Veuillen J-Y 2009 Phys. Rev. B 80235429

[40] Tsai M H, Chang C S, Dow J D and Tsong I S T 1992 Phys. Rev. B 451327

[41] Hoster H M, Kulakov M A and Bullemer B 1997 Surf. Sci. 382 L658

[42] Seubert A, Bernhardt J, Nerding M, Starke U and Heinz K 2000 Surf. Sci. 454-456 45

Seubert A, Saldin D K, Bernhardt J, Starke U and Heinz K 2000 J. Phys.: Condens. Matter 12 5527

[43] Hibino H, Kageshima H, Maeda F, NagaseM, Kobayashi Y and Yamaguchi H 2008 Phys. Rev. B 77075413

[44] Ohta T, El Gabaly F, Bostwick A, McChesney J L, Emtsev K V, Schmid A K, Seyller T, Horn K and Rotenberg E 2008 New J. Phys. 10023034

[45] Hiebel F, Mallet P, Varchon F, Magaud L and Veuillen J-Y 2009 Solid State Commun. 1491157

[46] Gu G, Nie S, Feenstra R M, Devaty R P, Choyke W J, Chan W K and Kane M G 2007 Appl. Phys. Lett. 90, 253507 
[47] Luxmi, Fishser P J, Srivastava N, Feenstra R M, Sun Y, Kedzierski J, Healey P and Gu G 2009 Appl. Phys. Lett. 95, 073101

[48] Emtsev K V et al 2009 Nature Mater. 8203

[49] Haas J, de Heer W A and Conrad E H 2008 J. Phys.: Condens. Matter 20323202

[50] Hannon J B and Tromp R M 2008 Phys. Rev. B 77, 241404(R)

[51] Virojanadara C, Syväjarvi M, Yakimova R, Johansson L I, Zakharov A A and Balasubramanian T 2008 Phys. Rev. B 78, 245403

[52] Haas J et al. 2008 Phys. Rev. Lett. 100125504

[53] Wiesendanger R 1994 Scanning Probe Microscopy and Spectroscopy (Cambridge: Cambridge University Press))

Chen C J 1993 Introduction to scanning Tunneling Microscopy (New York, NY: Oxford University Press)

[54] Kresse G and Hafner J 1993 Phys. Rev. B 47, 558

[55] Mallet P, Varchon F, Naud C, Magaud L, Berger C and Veuillen J-Y 2007 Phys. Rev. B 76 041403(R)

[56] Lauffer P, Emtsev K V, Graupner R, Seyller T, Ley L, Reshanov S A and Weber H B 2008 Phys. Rev. B 77155426

[57] Hembacher S, Giessibl F J, Mannhart J and Quate CF 2003 Proc. Natl Acad. Sci. USA 10012539

[58] Wang Y, Ye Y, Wu K 2006 Surf. Sci. 600729

[59] Varchon F, Mallet P, Magaud L and Veuillen J-Y 2008 Phys. Rev. B 77165415

[60] Li G, Luican A and Andrei E Y 2009 Phys. Rev. Lett. 102176804

[61] Rutter G M, Crain J N, Guisinger N P, First PN and Stroscio J A 2008 J. Vac. Sci. Technol. A 26938

[62] Yang H, Baffou G, Mayne A J, Comtet G, Dujardin G and Kuk Y 2008 Phys. Rev. B 78 $041408(\mathrm{R})$

[63] Stolyarova E et al 2007 PNAS 1049209

Zhang Y, Brar V W, Wang F, Girit C, Yayon Y, Panlasigui M, Zettl A and Crommie M F 2008 Nature Physics 4627

[64] Owman F and Martensson P 1996 Surf. Sci. 369126

Chen W et al. 2005 Surf. Sci. 596176

[65] Rutter G M, Guisinger N P, Crain J N, Jarvis E A A, Stiles M D, Li T, First P N and Stroscio J A 2007 Phys. Rev. B 76235416

[66] Kim S, Ihm J, Choi H J and Son Y-W 2008 Phys. Rev. Lett. 100176802

[67] Varchon F, Mallet P, Veuillen J-Y and Magaud L 2008 Phys. Rev. B 77235412

[68] Varchon F et al 2007 Phys. Rev. Lett. 99126805

[69] Mattausch A and Pankratov O 2007 Phys. Rev. Lett. 99076802 
[70] Borysiuk J, Bożek R, Strupiński W, Wysmołek A, Grodecki K, Stepniewski R and Baranowski J M 2009 J. Appl. Phys. 105, 023503

[71] Norimatsu W and Kusunoki M 2009 Chemical Physics Letters 46852

[72] Ohta T, Bostwick A, Seyller T, Horn K and Rotenberg E 2006 Science 313951

[73] Ohta T, Bostwick A, McChesney J L, Seyller T, Horn K and Rotenberg E 2007 Phys. Rev. Lett. 98206802

[74] Brar W V, Zhang Y, Yayon Y, Ohta T, McChesney J L, Bostwick A, Rotenberg E, Horn K and Crommie M F 2007 Appl. Phys. Lett. 91122102

[75] Vitali L, Riedl C, Ohmann R, Brihuega I, Starke U and Kern K 2008 Surf. Sci. 602 L127

[76] Crommie M F, Lutz C P and Eigler D M 1993 Nature (London) 363524

Hasegawa Y and Avouris P 1993 Phys. Rev. Lett. 711071

[77] Sprunger P T, Petersen L, Plummer E W, Laegsgaard E and Besenbacher F 1997 Science 275 1764

[78] Jeandupeux O, Burgi L, Hirstein A, Brune H and Kern K 1999 Phys. Rev. B 5915926.

[79] Kanisawa K, Butcher M J, Yamaguchi H and Hirayama Y 2001 Phys. Rev. Lett. 863384

[80] Pascual J I et al. 2004 Phys. Rev. Lett. 93196802

[81] Petersen L et al. 1998 Phys. Rev. B 57 R6858

[82] Brihuega I, Mallet P, Magaud L, Pons S, Custance O, Gomez-Rodrıguez J M and Veuillen J Y 2004 Phys. Rev. B 69155407

[83] Rutter G M, Crain J N, Guisinger N P, Li T, First P N and Stroscio J A 2007 Science 317, 219

[84] Mizes H A and Foster J S 1989 Science 244559

Kelly K F and Halas N J 1998 Surf. Sci. 416 L1085

Ruffieux P, Gröning O, Schwaller P, Schlapbach L and Gröning P 2000 Phys. Rev. Lett. 844910

Ruffieux P, Melle-Franco M, Gröning O, Bielmann M, Zerbetto F and Gröning P 2005 Phys. Rev. B 71153403

[85] Brihuega I et al. 2008 Phys. Rev. Lett. 101206802

[86] Peres N M R, Yang L and Tsai S-W 2009 New. J. Phys. 11095007

[87] Bena C 2008 Phys. Rev. Lett. 100076601

[88] Pereg-Barnea T and McDonald A H 2008 Phys. Rev. B 78014201

[89] Bena C 2009 Phys. Rev. B 79125427

[90] Simon L, Bena C, Vonau F, Aubel D, Nasrallah H, Habar M and Peruchetti J C 2009 Eur. Phys. J. B 69351

[91] Al-Temimy A, Riedl C and Starke U 2009 Appl. Phys. Lett. 95231907

[92] Camara N, Huntzinger J-R, Rius G, Tiberj A, Mestres N, Pérez-Murano F, Godignon P and Camassel J 2009 Phys. Rev. B 80125410 
[93] Wu X, Hu Y, Ruan M, Madiomanana N K, Hankinson J, Sprinkle M, Berger C and de Heer W A 2009 Appl. Phys. Lett. 95223108

[94] Magaud L, Hiebel F, Varchon F, Mallet P and Veuillen J-Y 2009 Phys. Rev. B 79 161405(R)

[95] Magaud L, Hiebel F, Varchon F, Mallet P and Veuillen J-Y 2009 Phys. Status Solidi RRL 3172 


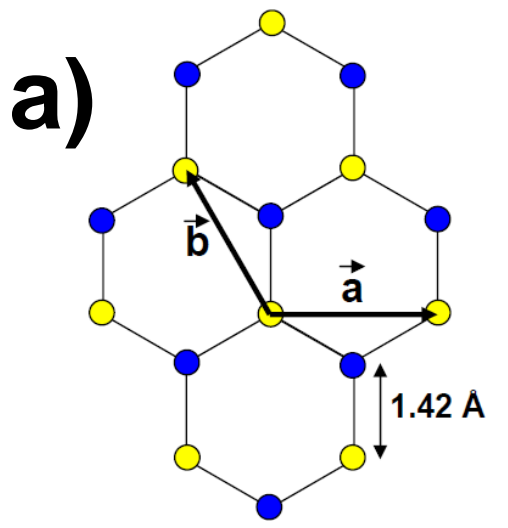

A atoms

B atoms

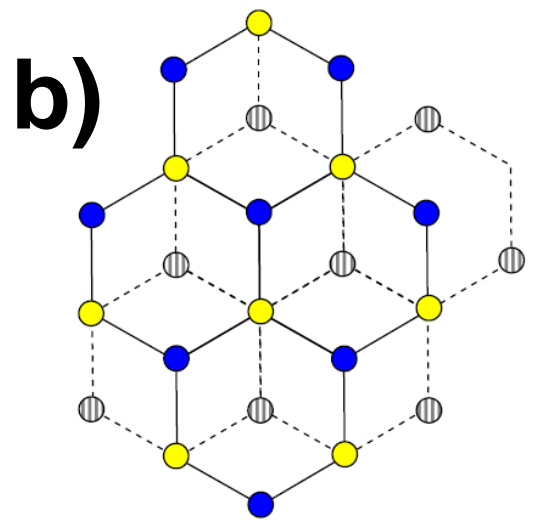

A atoms top layer

B atoms top layer

(11) A atoms $2^{\text {nd }}$ layer
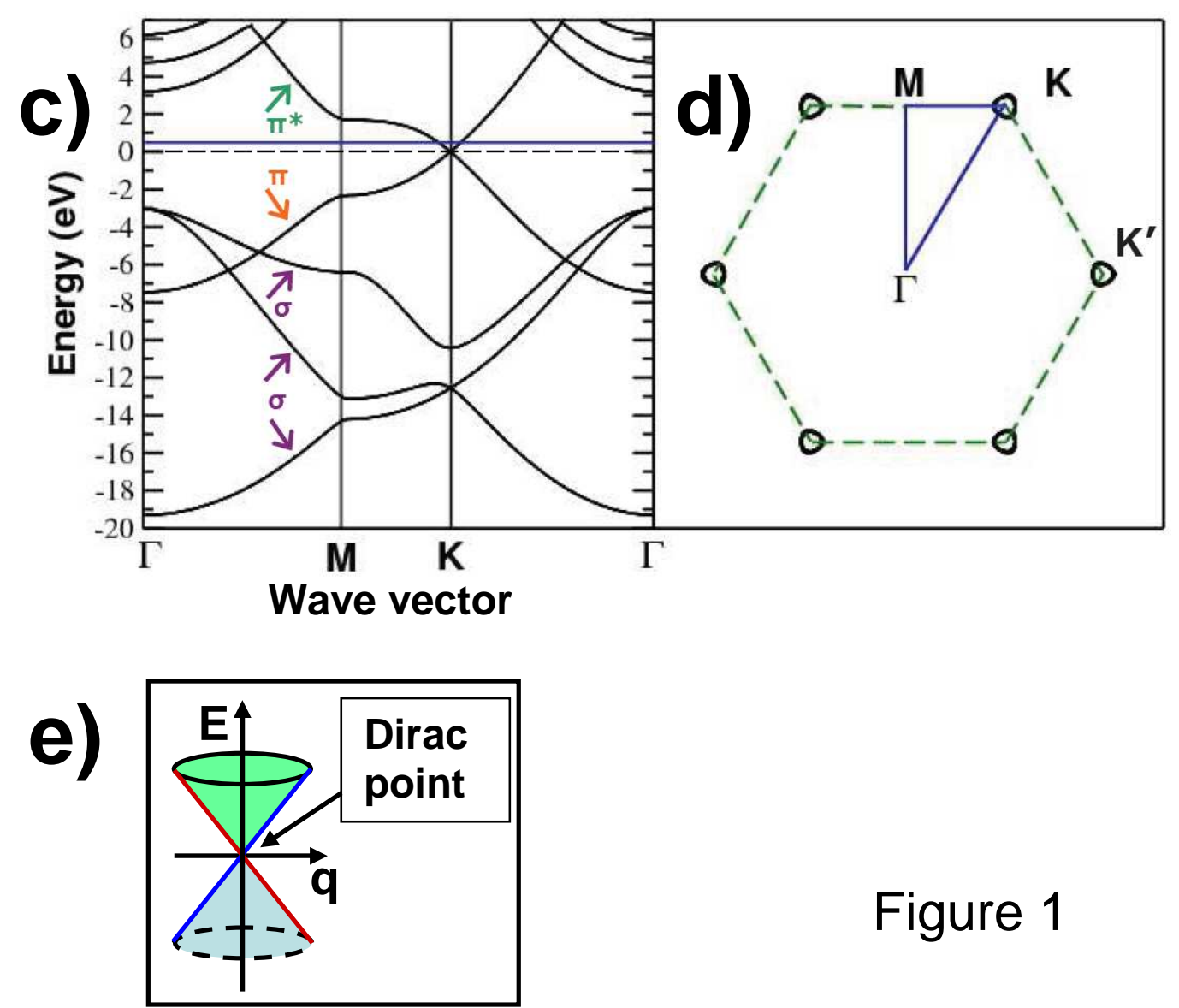

Figure 1

Figure 1: (a): schematic (top) view of the atomic structure of monolayer graphene highlighting the two atomic sublattices (A and B). (b): top view of the atomic structure of bilayer graphene with Bernal stacking. (c) band structure of monolayer graphene obtained from ab-initio calculations. The nature of the bands $(\sigma$ or $\pi$ ) is indicated. (d): green dashed lines: first Brillouin zone (BZ) of graphene; black lines: isoenergy contours for an energy $+0.5 \mathrm{eV}$ above the Dirac point. (e): « Dirac cones » representing the low energy band structure of graphene close to the K (K') points of the BZ. From [21]. 

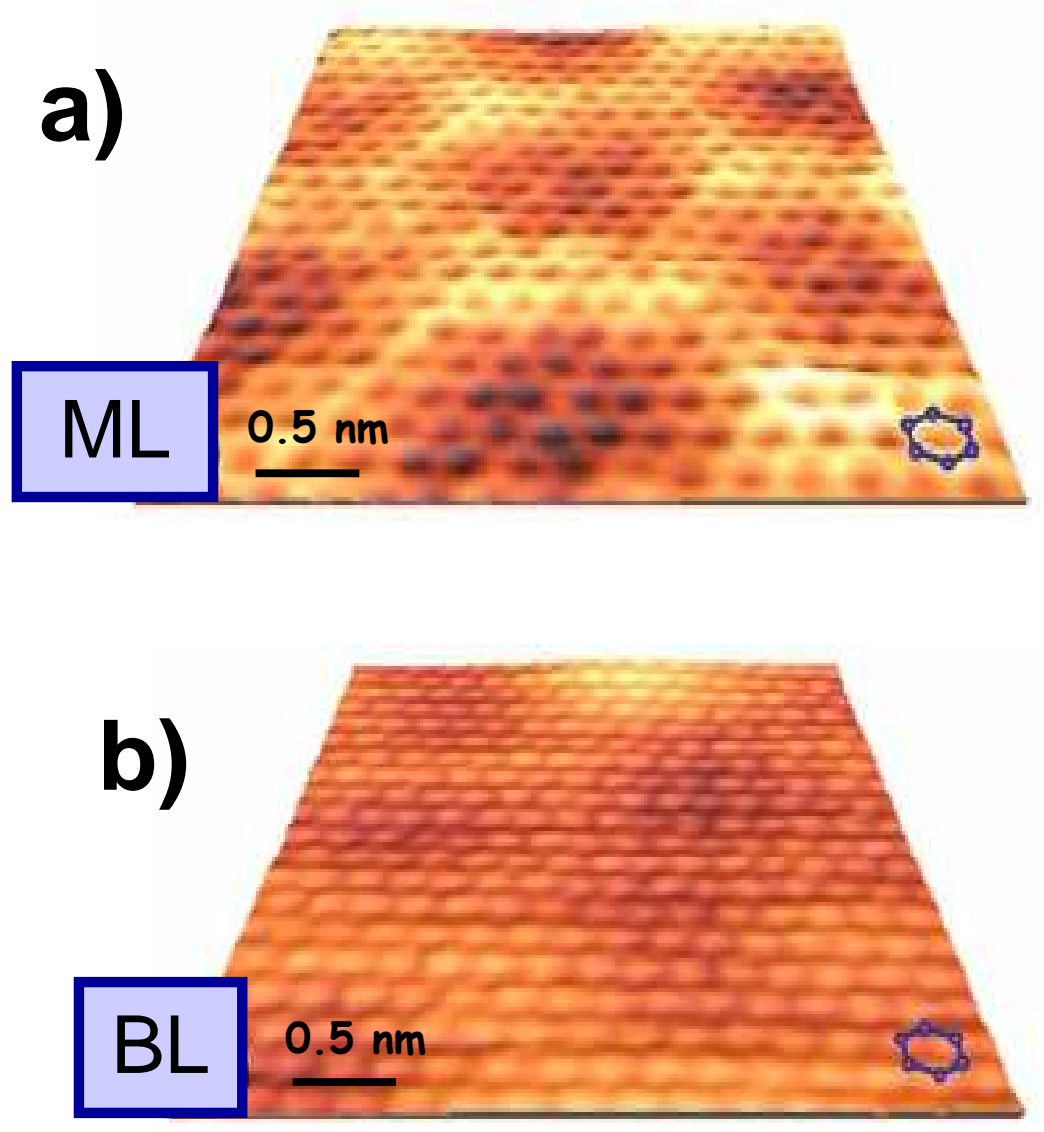

Figure 2: Low bias images of graphene monolayer (a) and bilayer (b). Size of the images: $4 \mathrm{x} 4 \mathrm{~nm}^{2}$; sample bias: $+200 \mathrm{mV}$. From [55]. Copyright 2007 by the American Physical Society.

\section{Figure 2}



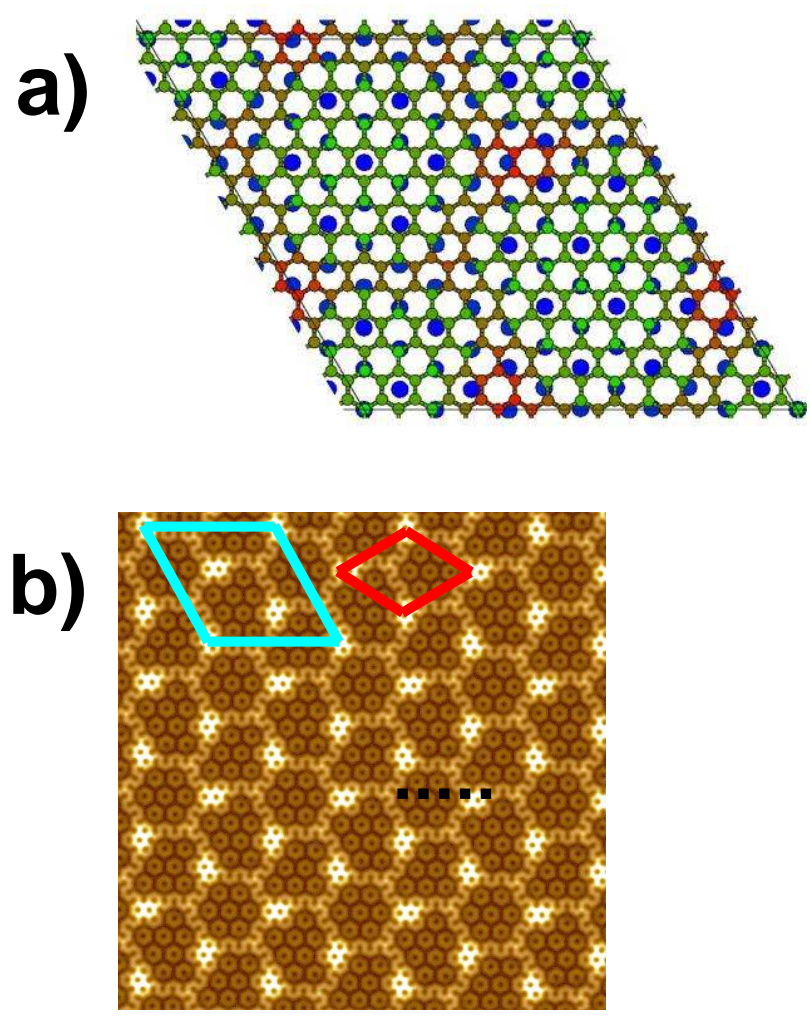

\section{Figure 3}

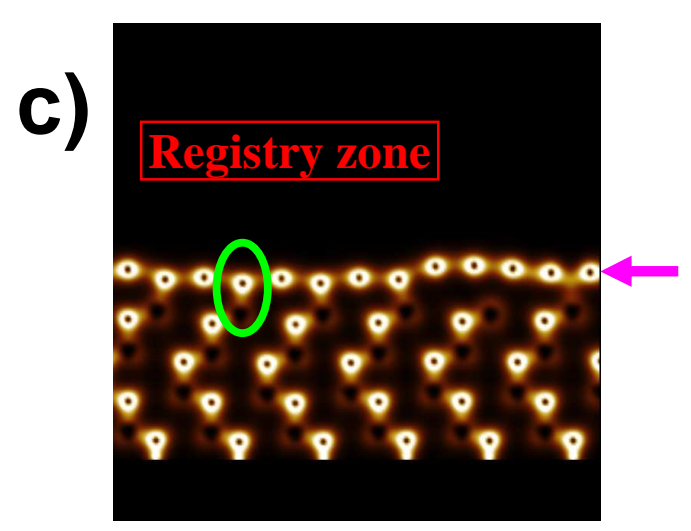

Figure 3: (a) Relaxed 6R3 cell for $1 \mathrm{C}$ plane on top of the $\mathrm{SiC}$ surface. The atoms of the last $\mathrm{Si}$ plane of $\mathrm{SiC}$ (large blue circles) and of the graphitic layer (small red to green circles) are shown. The color of the $\mathrm{C}$ atom are related to their position on top of $\mathrm{SiC}$, low : green, high : red. (b, c) $\mathrm{Ab}$ initio calculation (total charge density) of the atomic structure of the first $\mathrm{C}$ layer on the $\mathrm{Si}$ face of SiC. (b) : top view just above the $\mathrm{C}$ plane, (c) : cross section along the dashed black line shown in (b). The green oval in (c) indicate a spot (located in the registry zone) where a chemical bond is formed between the $\mathrm{C}$ plane (purple arrow) and the substrate. From [67]. Copyright 2008 by the American Physical Society. 


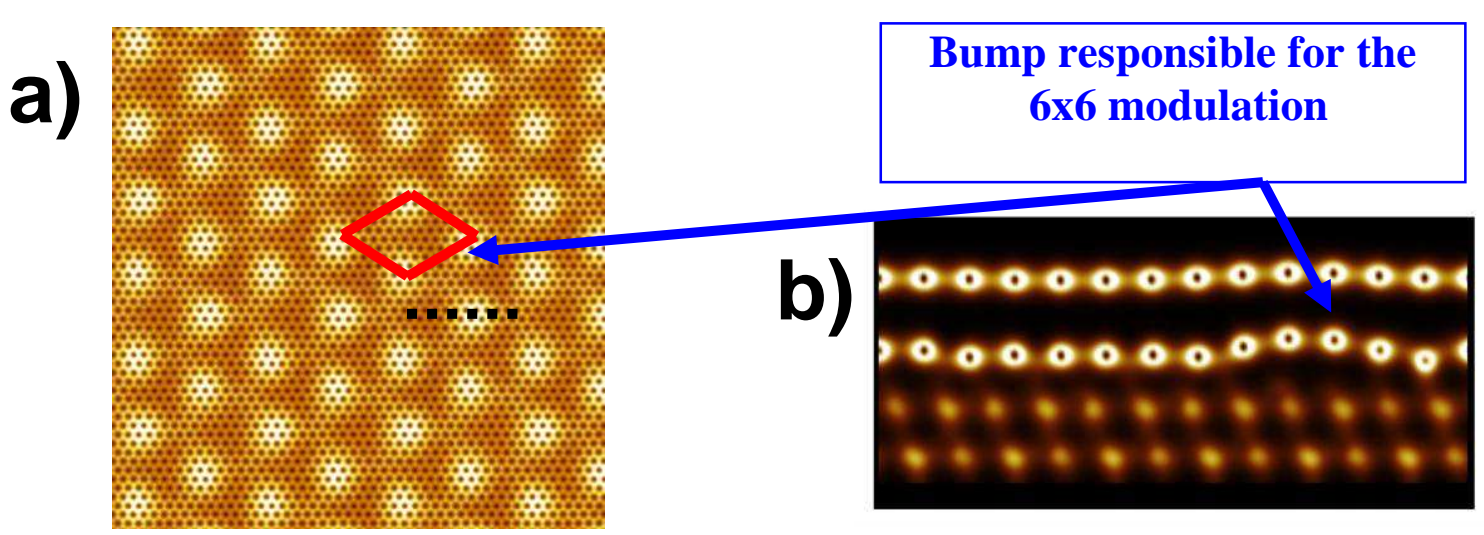

Figure $4:$ ab initio calculation (total charge density) of the atomic structure of two $\mathrm{C}$ layers on the $\mathrm{Si}$ face of $\mathrm{SiC}$. (a) : top view just above the surface $\mathrm{C}$ plane, the red diamond indicates a (6x6) unit cell. (b) : cross section along the dashed black line shown in a. From [67]. Copyright 2008 by the American Physical Society.

\section{Figure 4}

\section{a)}

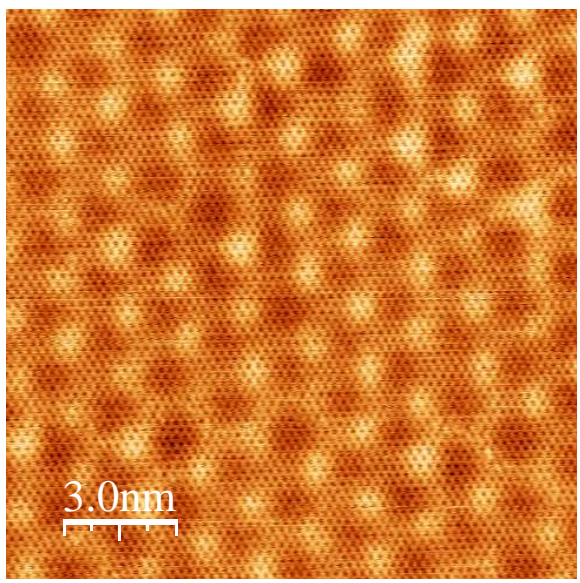

b)

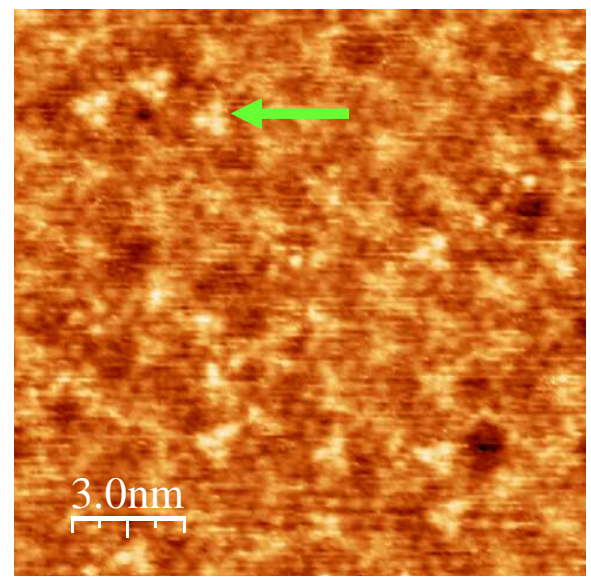

Figure 5: STM images of monolayer graphene on $\mathrm{SiC}(0001)$, size: $15 \times 15 \mathrm{~nm}^{2}$.

The same domain is imaged at two different sample biases; (a): $-0.03 \mathrm{~V},(\mathrm{~b}):-0.5 \mathrm{~V}$.

High (low) bias image in (b) ((a)) reveals the interface (graphene) structure. The location of a special interface feature discussed in the text is indicated by an arrow.

\section{Figure 5}



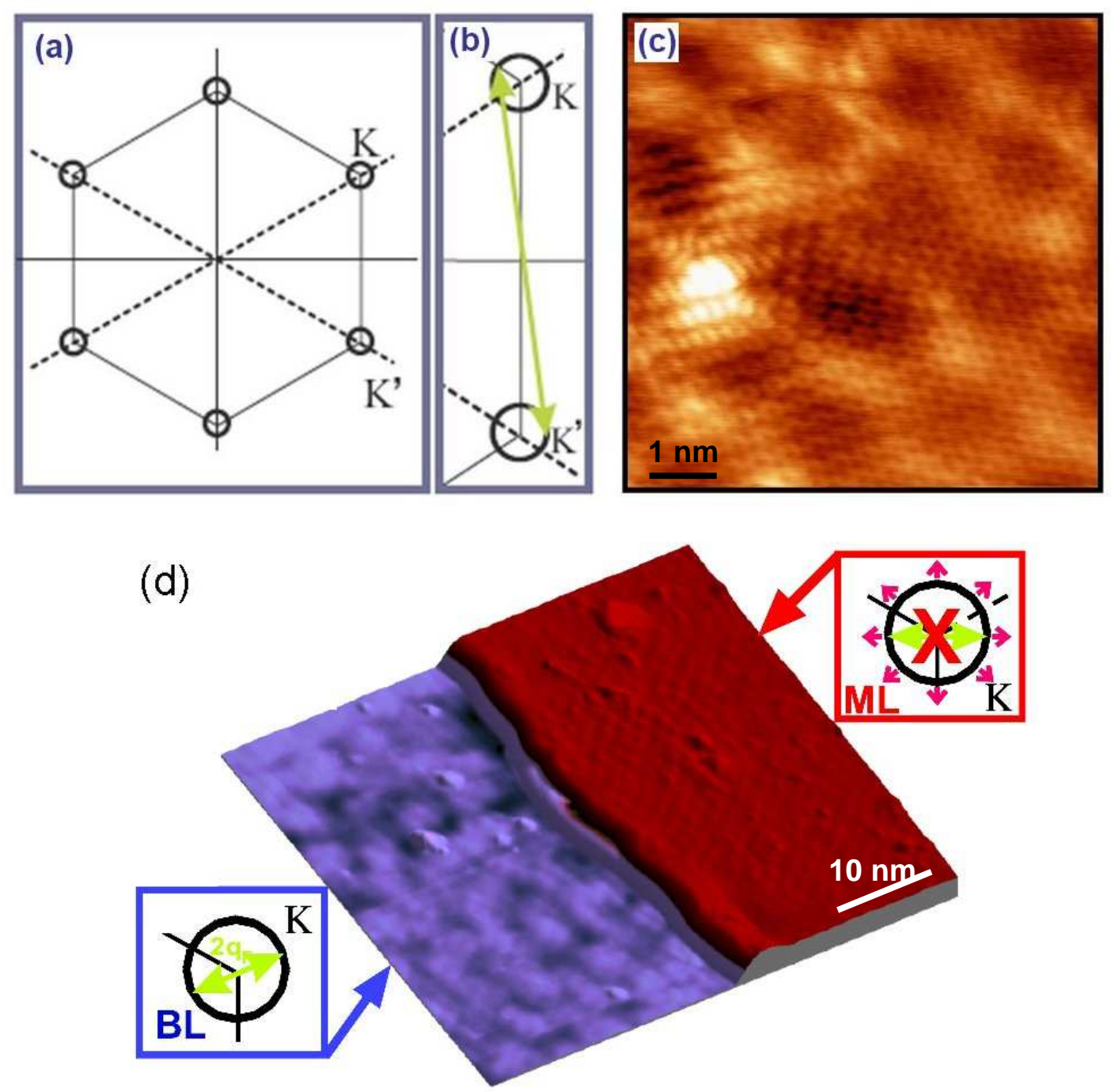

Figure 6. (a) Schematic Fermi surface of monolayer and bilayer graphene, derived from ARPES measurements $[13,72]$. It is built on two circular pockets of radius $\mathrm{q}_{\mathrm{F}}$ centered at $\mathrm{K}$ and $\mathrm{K}$ ' points. (b) Illustration of an intervalley scattering process. (c) Low bias $7 \times 7 \mathrm{~nm}^{2}$ constant current image of monolayer graphene, including an atomic-sized impurity. Quasiparticles elastic scattering off the impurity generates a periodic modulation of the LDOS, the so-called $(\sqrt{3} \mathrm{x} \sqrt{3}) \mathrm{R} 30^{\circ}$ superstructure, which is inferred to the intervalley scattering processes. Sample bias: $-100 \mathrm{mV}$. From [55]. (d) 3D rendered $50 \times 50 \mathrm{~nm} 2 \mathrm{STM}$ image of two adjacent monolayer and bilayer graphene terraces. The image was recorded at $4 \mathrm{~K}$, at sample bias $+1 \mathrm{mV}$. A long period $(\sim 5.2 \mathrm{~nm})$ superstructure is found on bilayer graphene (left terrace), corresponding to the intravalley backscattering processes sketched in the left box. This superstructure is not observed on monolayer graphene (right terrace), a consequence of electronic chirality (see text).

Image recorded in the group of Prof. K. Kern, MPI Stuttgart. From [85]. Copyright 2008 by the American Physical Society

\section{Figure 6}



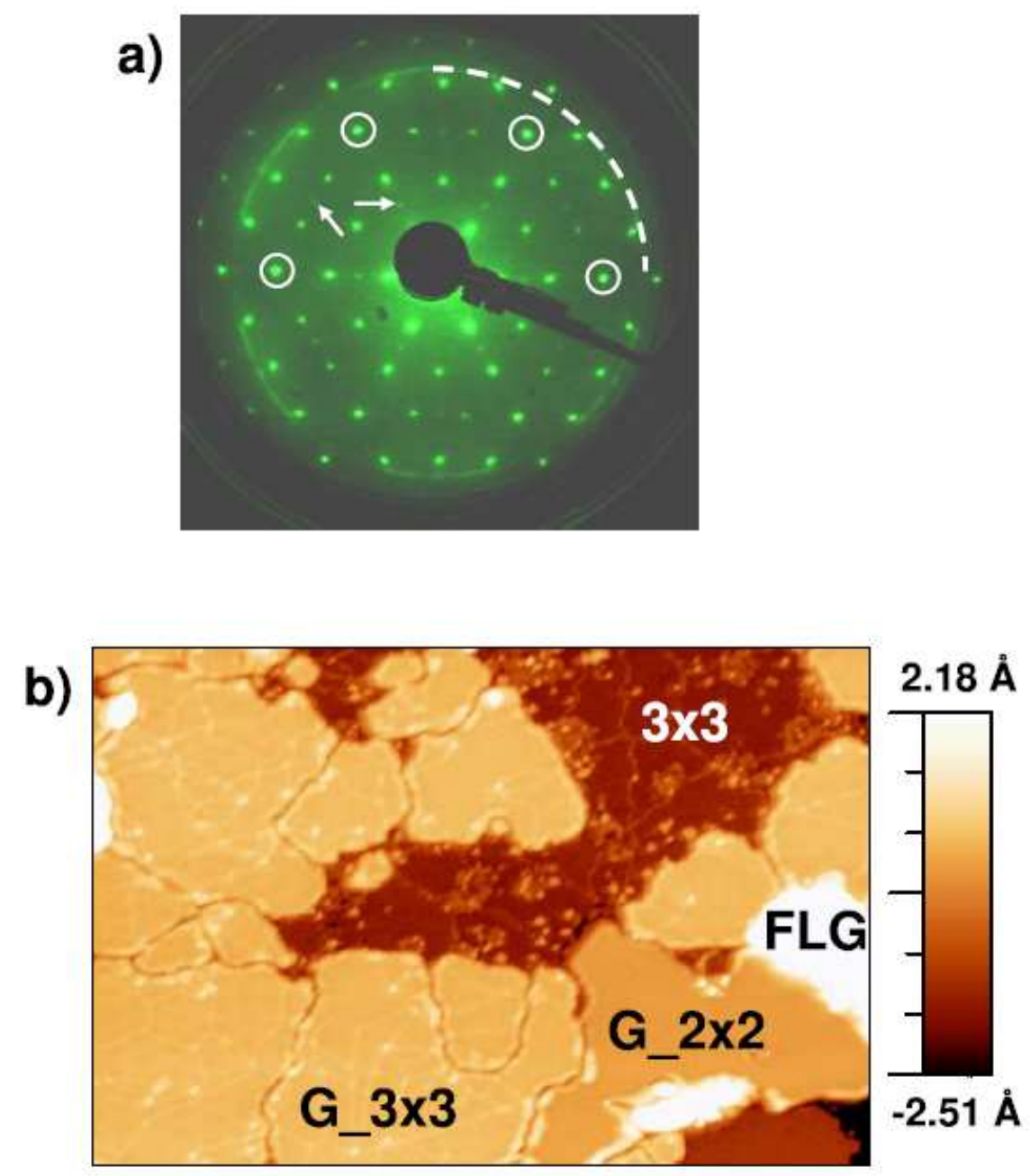

Figure 7: (a) LEED pattern of a lightly graphitized $\mathrm{C}$ face $\mathrm{SiC}$ substrate. The average graphene coverage is less than one monolayer. The circles indicate the $\mathrm{SiC}(1 \mathrm{x} 1)$ spots, the arrows point to the spots of the $(2 \times 2)$ reconstruction of the $\mathrm{SiC}$ surface and the dashed (quarter) circle indicates the location of the graphene related signal (incomplete ring). (b) STM image of the same sample, size: $120 \times 80 \mathrm{~nm}^{2}$, sample bias: $+2.5 \mathrm{~V}$. The dark areas labelled « $3 \times 3$ » correspond to the bare $\mathrm{SiC}$ surface with $(3 \times 3)$ reconstruction. Areas labelled G_3x3 and G_2x2 correspond to the two kinds of monolayer graphene islands discussed in the text. From [38]. Copyright 2008 by the American Physical Society.

\section{Figure 7}



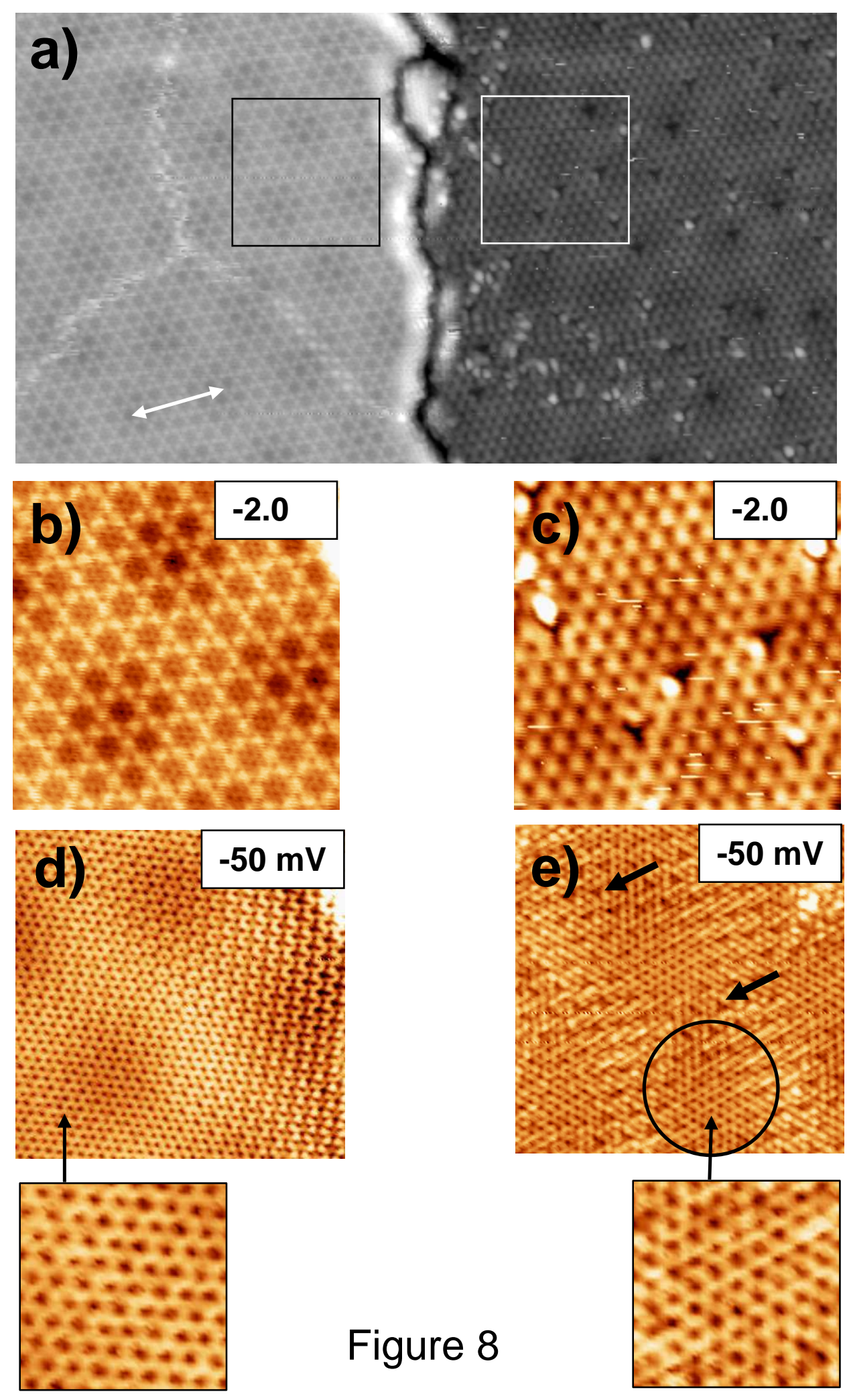
$-50 \mathrm{mV}$
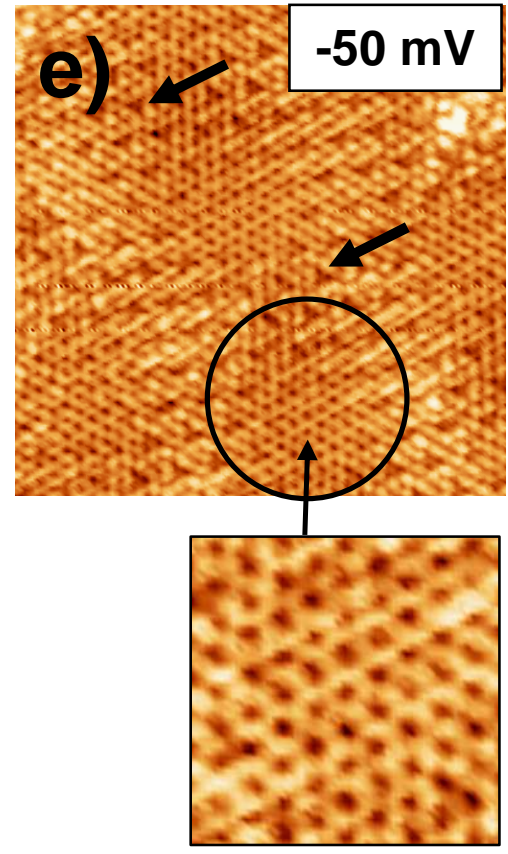

Figure 8 
Figure 8: (a) lmage of a boundary between a $G \_3 \times 3$ island (left) and a $G \_2 \times 2$ island (right). image size: $40 \times 22 \mathrm{~nm}^{2}$, sample bias: $-2.0 \mathrm{~V}$. The double-sided arrows indicate the superstructure discussed in 3.2 (b) and (d): zoomed-in high and low bias images on the G_3x3 island of (a) (boxed area), size: $7.5 \times 7.5 \mathrm{~nm}^{2}$. (c) and (e): zoomed-in high and low bias images on the G_2x2 island of (a) (boxed area), size: $7.5 \times 7.5 \mathrm{~nm}^{2}$. Sample bias: $-2.0 \mathrm{~V}$ for (b) and (c), $-50 \mathrm{mV}$ for (d) and (e). The lower frames are zoomed-in images on (d) and (e) which reveal the honeycomb contrast, size: $2 \times 2 \mathrm{~nm}^{2}$. Arrows in (e) indicate the structures due to graphene-substrate interaction which appear as «missing atoms », see text. From [38]. Copyright 2008 by the American Physical Society.

\section{Figure 8 continued}

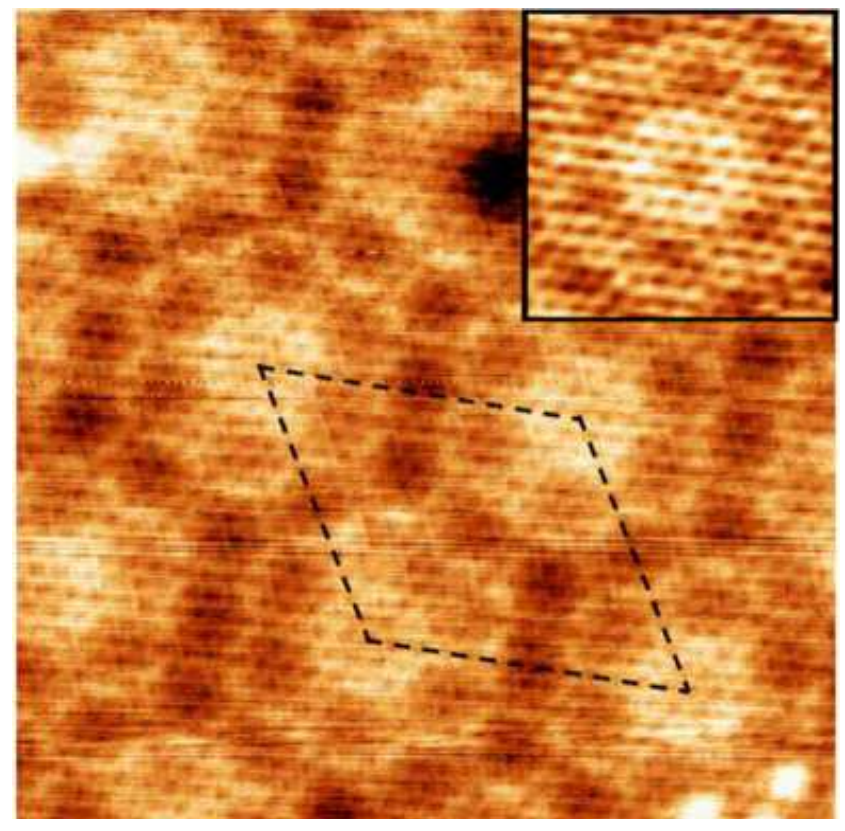

Figure 9: $8 \times 8 \mathrm{~nm}^{2}$ STM images of a G_3x3 island where the graphene layer is rotated by $30^{\circ}$ with respect to the $\mathrm{SiC}$ lattice, sample bias: $-1.5 \mathrm{~V}$. The $\mathrm{SiC} 6 \mathrm{R} 3$ common cell is represented with dashed lines. Insert: $3 \times 3 \mathrm{~nm}^{2} \mathrm{STM}$ image of the same island; sample bias: $+100 \mathrm{mV}$. The graphene layer shows an AB symmetric honeycomb contrast. From [39]. Copyright 20097 by the American Physical Society.

\section{Figure 9}




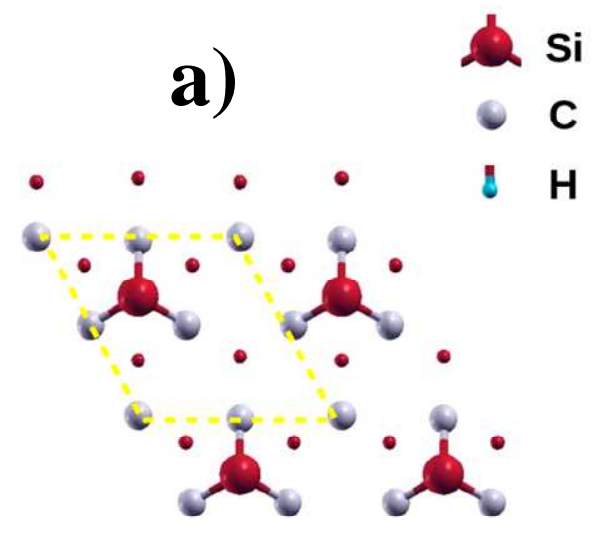

b)
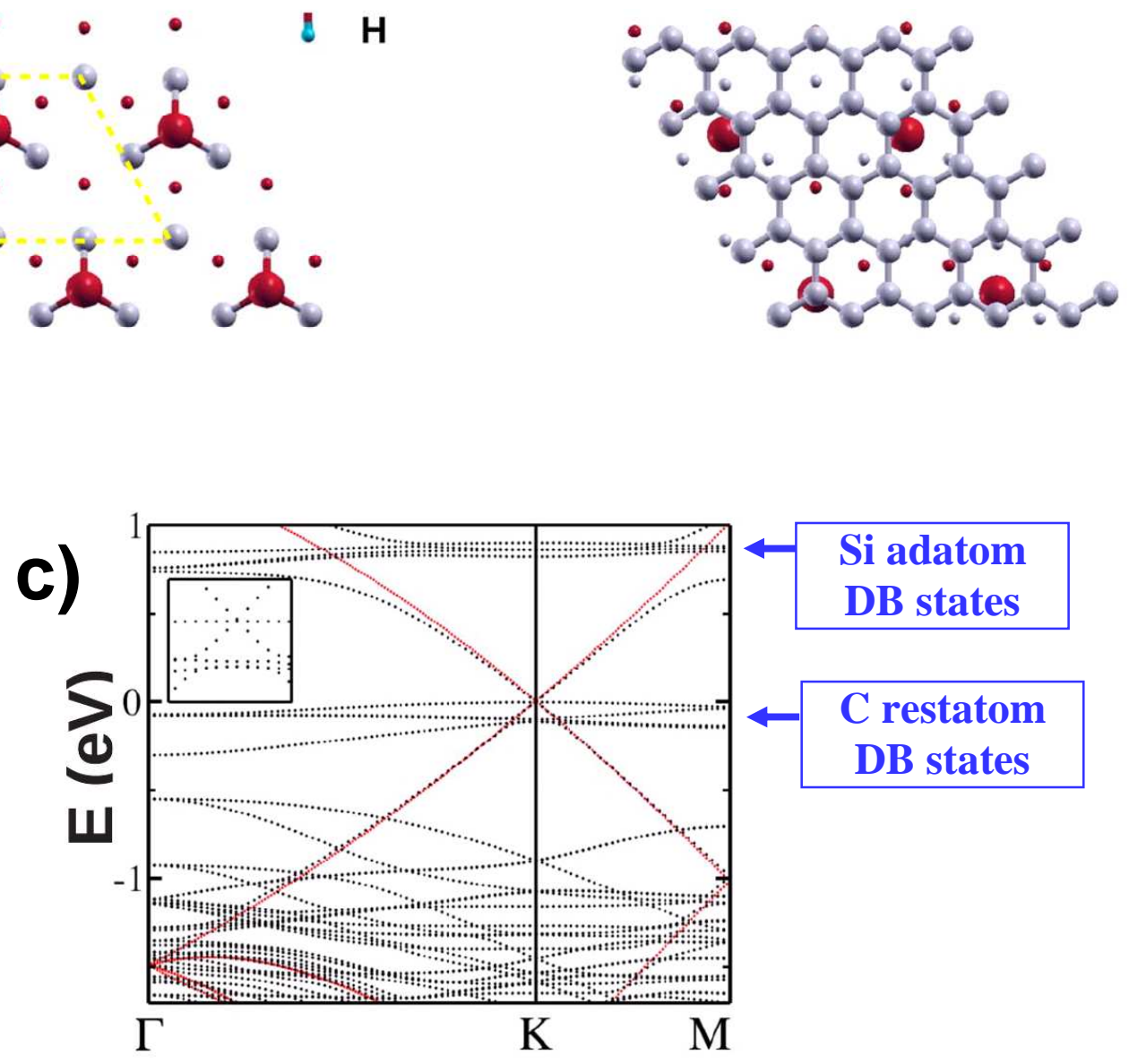

Figure 10 : (a) : geometry of the bare $(2 \times 2)_{C}$ reconstruction of the C-terminated SiC surface. The $2 \times 2$ cell is shown in yellow. The silicon atom (red) radii are related to their height with respect to $\mathrm{SiC}$ - the larger, the higher. (b) : geometry of the graphene on the same $(2 \times 2)_{\mathrm{C}}$ reconstruction. A common $(4 \mathrm{x} 4)$ cell is shown. (c) Band structure of a graphene layer on top of the $(2 \times 2)_{C}$ reconstruction (in the configuration of figure (b)) that shows the linear dispersion characteristic of graphene at low energy. The weakly dispersing bands around 0 and $0.8 \mathrm{eV}$ are related to dangling bonds $(\mathrm{DB})$ states of the $(2 \times 2)_{\mathrm{C}}$ surface reconstruction. From [94]. Copyright 20097 by the American Physical Society.

\section{Figure 10}

